\title{
EXAMPLES OF POINTED COLOR HOPF ALGEBRAS
}

\author{
NICOLÁS ANDRUSKIEWITSCH, IVÁN ANGIONO, DIRCEU BAGIO
}

\begin{abstract}
We present examples of color Hopf algebras, i. e. Hopf algebras in color categories (braided tensor categories with braiding induced by a bicharacter on an abelian group), related with quantum doubles of pointed Hopf algebras. We also discuss semisimple color Hopf algebras.
\end{abstract}

\section{INTRODUCTION}

Color Lie algebras appeared in $\mathrm{Re}$ (under the name generalized Lie algebra of type $\chi$ ), were rediscovered in [S] and then studied in several papers, e. g. $\mathrm{BaP}, \mathrm{Hu}, \mathrm{Ky}, \mathrm{CSO}, \mathrm{ScZ}, \mathrm{CPO}, \mathrm{Mo}$. A color Lie algebra is a Lie algebra in a symmetric tensor category, namely the category of $G$-graded vector spaces over a finite abelian group $G$ with symmetry given by a skewsymmetric bicharacter $\beta: G \times G \rightarrow \mathbb{k}^{\times}$. Hopf algebras, unlike Lie algebras, can be defined in braided tensor categories. In this paper we study Hopf algebras in the braided tensor category of $G$-graded vector spaces with braiding given by a (not necessarily skew-symmetric) bicharacter $\beta: G \times G \rightarrow \mathbb{k}^{\times}$: we call them color Hopf algebras. Pointed Hopf superalgebras were considered in [AAY] in relation with the classification of finite-dimensional Nichols algebras of diagonal type [H1. However some of the Nichols algebras in the list of [H1] are neither of standard (close to simple Lie algebras) nor of super type. We wonder whether they are related with color Hopf algebras, and this is one of the motivations of the present article, whose contents we describe next. Section 1 is devoted to basic constructions and results in color categories; albeit these are particular instances of constructions and results in braided tensor categories, we feel that the explicit formulae in this context might be useful for the reader. We also relate the symmetric color categories with the classification of semisimple triangular Hopf algebras over a algebraically closed field EG1, EG2]. In Section 2, we adapt the lifting method [AS] to the setting of color Hopf algebras. In Section 3, we produce color Hopf algebras versions of the quantum doubles of bosonizations of color Nichols algebras. We introduce the notion of consistent coloring of a generalized Dynkin diagram, meaning that is preserved by the movements of the Weyl groupoid. In Section 4, we discuss some examples of semisimple color Hopf algebras.

2000 Mathematics Subject Classification. 16T05,17B75.

N. A. and I. A. were partially supported by CONICET, FONCyT-ANPCyT, Secyt (UNC).

D. B. was supported through CNPq - 201586/2010-0, CAPES-MATH-AmSud. 


\section{Preliminaries and NOtation}

Let $\mathbb{k}$ be an algebraically closed field of characteristic 0 and $\mathbb{k}^{\times}=\mathbb{k}-\{0\}$; all vector spaces, algebras and tensor products are over $\mathbb{k}$.

Throughout this paper, $G$ is a finite abelian group, denoted multiplicatively, and $A=\widehat{G}=\operatorname{Hom}\left(G, \mathbb{k}^{\times}\right)$is the character group of $G$. Then $\left(e_{g}\right)_{g \in G}$ denotes the canonical base of $\mathbb{k} G$ and Aut $G$ the group of automorphisms of $G$. The elements of the dual basis of $(\mathbb{k} G)^{*}=\mathbb{k}^{G}$ are denoted by $\delta_{g}$, $g \in G$. Let $L$ be an abelian group and suppose that $G$ acts on $L$; we denote by $Z^{1}(G, L)$ (resp. $Z^{2}(G, L)$ ) the set of normalized 1-cocycles (resp. 2-cocycles). If $A$ acts on a set $X$ and $x \in X$, then $\operatorname{Stab}_{A}(X)$ is the stabilizer of the action and $A^{x}$ is the isotropy subgroup of $x . C_{N}, N \in \mathbb{N}$, is the cyclic group of order $N$. For an arbitrary group $\Gamma, Z(\Gamma)$ denotes its center.

We also denote by $G(C)$ the set of all group-like elements of a coalgebra $C$. The category of left, respectively right, $C$-comodules is denoted by ${ }^{C} \mathcal{M}$; resp. $\mathcal{M}^{C}$. If $B$ is an algebra, then the category of left, respectively right, $B$-modules is denoted by ${ }_{B} \mathcal{M}$; resp. $\mathcal{M}_{B}$. When $B$ is a bialgebra, $\mathcal{P}(B)$ denotes the set of all primitive elements.

All Hopf algebras in this paper have bijective antipodes. Let $H$ be a Hopf algebra. We will denote by Aut $H$ the group of Hopf algebra automorphisms of $H$; and by $\mathcal{H} z(H)$ the Hopf center of $H$, i. e., the maximal central Hopf subalgebra of $H$ [A]. We assume that the reader has familiarity with tensor categories, see e. g. [BK], and with Yetter-Drinfeld modules and bosonization, see e. g. [AS]. Recall that a tensor functor between tensor categories $\mathcal{C}$ and $\mathcal{D}$ (with associativity $a$ ) is a pair $(F, \phi)$, where $F: \mathcal{C} \rightarrow \mathcal{D}$ is an additive $\mathbb{k}$-linear functor and $\phi_{X, Y}: F(X \otimes Y) \rightarrow F(X) \otimes F(Y)$ is a natural isomorphism such that $a_{F(X), F(Y), F(Z)}\left(\phi_{X, Y} \otimes \mathrm{id}\right) \phi_{X \otimes Y, Z}=$ (id $\left.\otimes \phi_{Y, Z}\right) \phi_{X, Y \otimes Z} F\left(a_{X, Y, Z}\right)$ for all $X, Y, Z \in \mathcal{C}$, and some compatibility for the unit. If $\mathcal{C}$ and $\mathcal{D}$ are braided (with braiding $c$ ), a monoidal tensor functor $F: \mathcal{C} \rightarrow \mathcal{D}$ is braided when for all $X, Y \in \mathcal{C}$

$$
c_{F(X), F(Y)} \phi_{X, Y}=\phi_{Y, X} F\left(c_{X, Y}\right) .
$$

1.1. Color categories. We denote by $\operatorname{Vect}^{G}$ the category of $G$-graded vector spaces. Let $V=\oplus_{g \in G} V_{g} \in \operatorname{Vect}^{G}$ and $v \in V$. If $v \in V_{g}$, then we write $|v|=g$. The support of $V$ is $\sup V=\left\{g \in G: V_{g} \neq 0\right\}$. If $v=\sum_{g \in G} v_{g}$, then the support of $v$ is $\sup v=s(v)=\left\{g \in G: v_{g} \neq 0\right\}$. The category $\operatorname{Vect}^{G}$ is a tensor one: if $V, W \in \operatorname{Vect}^{G}$, then $V \otimes W \in \operatorname{Vect}^{G}$ with $(V \otimes W)_{g}=\oplus_{h l=g} V_{h} \otimes W_{l}$. Let $\mathbb{k}_{g} \in \operatorname{Vect}^{G}, g \in G$ be given by $\left(\mathbb{k}_{g}\right)_{l}=\mathbb{k}$ if $l=g$ and $\left(\mathbb{k}_{g}\right)_{l}=0$ otherwise. Thus any object is a direct sum of copies of various $\mathbb{k}_{g}$. Then $\mathbb{k}_{g} \otimes \mathbb{k}_{h} \simeq \mathbb{k}_{g h}$. The unit object is $\mathbb{k}=\mathbb{k}_{1}$. The full subcategory vect ${ }^{G}$ of finite dimensional $G$-graded vector spaces is rigid: if $V \in \operatorname{vect}^{G}$, then $V^{*}=\operatorname{Hom}(V, \mathbb{k})=\oplus_{g \in G} V_{g}^{*}$, with $V_{g}^{*}=\operatorname{Hom}\left(V_{g^{-1}}, \mathbb{k}\right)$.

A bicharacter on $G$ is a $\mathbb{Z}$-bilinear form $\beta: G \times G \rightarrow \mathbb{K}^{\times}$, that is,

$$
\beta(g h, l)=\beta(g, l) \beta(h, l), \quad \beta(g, h l)=\beta(g, h) \beta(g, l), \quad g, h, l \in G .
$$


A commutation factor on $G$ is a skew-symmetric bicharacter $\beta$, that is

$$
\beta(g, h) \beta(h, g)=1, \quad g, h \in G .
$$

Let $\beta$ be a bicharacter on $G$. Then $\operatorname{Vect}^{G}$ is braided with braiding given by $c=c_{\beta}: V \otimes W \rightarrow W \otimes V, \quad c(v \otimes w)=\beta(g, h) w \otimes v, \quad v \in V_{g}, \quad w \in W_{h}$.

Here it is crucial that $G$ is abelian, for $c$ to be a map of $G$-graded vector spaces. Conversely, any braiding in the tensor category $\operatorname{Vect}^{G}$ comes from a bicharacter $\beta$ on $G$. Furthermore, $c_{\beta}$ is a symmetry in $\operatorname{Vect}^{G}$ if and only if $\beta$ is a commutation factor on $G$.

Definition 1.1. Let $G$ be a finite abelian group and $\beta$ a bicharacter on $G$. We denote by $\operatorname{Vect}_{\beta}^{G}$ the braided tensor category $\operatorname{Vect}^{G}$ with braiding $c_{\beta}$. Any braided tensor category like this is called a color category with color $\beta$.

Remark 1.2. (i). Let $G, \Gamma$ be finite abelian groups, $\beta$ and $\vartheta$ bicharacters on $G$ and $\Gamma$ respectively, $\psi: G \rightarrow \Gamma$ a group homomorphism and $\gamma: G \times G \rightarrow \mathbb{k}^{\times}$a normalized 2-cocycle. Then there is a tensor functor $(F, \phi): \operatorname{Vect}^{G} \rightarrow \operatorname{Vect}^{\Gamma}$ given by $F\left(\mathbb{k}_{g}\right)=\mathbb{k}_{\psi(g)}, \phi_{\mathbb{k}_{g}, \mathbb{k}_{h}}=\gamma(g, h)$. Assume that for all $g, h \in G$

$$
\beta(g, h)=\vartheta(\psi(g), \psi(h)) \frac{\gamma(g, h)}{\gamma(h, g)} .
$$

Then $(F, \phi): \operatorname{Vect}_{\beta}^{G} \rightarrow \operatorname{Vect}_{\vartheta}^{\Gamma}$ is a braided tensor functor, see (1.1).

(ii) Let $\vartheta:(A \times G) \times(A \times G) \rightarrow \mathbb{k}^{\times}$be given by $\vartheta((\xi, g),(\eta, h))=\eta(g)$. Then $\operatorname{Vect}_{\vartheta}^{A \times G}$ and $\mathbb{k}_{\mathbb{k} G}{ }^{\mathbb{k}} y \mathcal{D}$ are equivalent braided tensor categories.

(iii) The color category $\operatorname{Vect}_{\beta}^{G}$ is actually isomorphic to a braided subcategory of $\mathbb{k}_{\mathbb{k}}^{\mathbb{k}} G \mathcal{D}$. Indeed, let $\chi, \chi^{o}: G \rightarrow A$ be given by

$$
\chi_{g}(h)=\beta(h, g)=\chi_{h}^{o}(g), \quad g, h \in G .
$$

Then the morphism $G \rightarrow A \times G, g \mapsto\left(\chi_{g}, g\right)$, together the trivial cocycle, induce a braided tensor functor $\operatorname{Vect}_{\beta}^{G} \rightarrow \operatorname{Vect}_{\vartheta}^{A \times G} \simeq \mathbb{k}_{\mathbb{k} G} G \mathcal{D}$. Explicitly, if $V \in \operatorname{Vect}^{G}$, then $V \in \in_{\mathbb{R} G}^{\mathbb{R} G} y \mathcal{D}$ with action and coaction

$$
e_{g} \cdot v=\beta(g,|v|) v, \quad \delta(v)=e_{|v|} \otimes v, \quad v \in V, g \in G .
$$

In what follows, we fix a bicharacter $\beta$ and use the adjective graded for notions in $\operatorname{Vect}^{G}$, that do not require the braiding, and color for notions in $\operatorname{Vect}_{\beta}^{G}$, that do.

1.2. Graded algebras. A $G$-graded algebra is the same as an associative algebra in $\operatorname{Vect}^{G}$. If $V \in \operatorname{vect}^{G}$, then $\operatorname{End}(V)$ is a graded algebra with $\operatorname{End}(V)_{g}=\left\{T \in \operatorname{End}(V): T\left(V_{h}\right) \subset V_{g h}\right.$, for all $\left.h \in G\right\}$, for all $g \in G$. A graded representation of a $G$-graded algebra $B$ on $V \in$ vect $^{G}$ is a $G$-graded morphism $\rho: B \rightarrow \operatorname{End}(V)$; for general $V$, a graded representation is a morphism into the subalgebra $\mathcal{E N D}(V)=\oplus_{g \in G} \operatorname{End}(V)_{g}$ of $\operatorname{End}(V)$. Note that $\rho: B \rightarrow \operatorname{End}(V)$ is a graded representation if and only if there is an 
action $B \otimes V \rightarrow V$ that respects the grading. In this case, we say that $V$ is a left graded module. Analogously, there are right graded modules. A graded bimodule is a bimodule such that both the left and the right actions are homogeneous. The respective abelian categories are denoted by ${ }_{B} \mathcal{G M}$, $\mathcal{G M}_{B}$ and ${ }_{B} \mathcal{G M}_{B}$, with obvious morphisms.

Remark 1.3. Let $B$ be a graded algebra and $B_{G}=B \# \mathbb{k} G$ the smash product algebra; this is the vector space $B \otimes \mathbb{k} G$ with the multiplication given by

$$
\left(x \# e_{g}\right)\left(y \# e_{h}\right)=\beta(g,|y|) x y \# e_{g h}, \quad x, y \in B, \quad g, h \in G .
$$

Note that if $V \in{ }_{B} \mathcal{G M}$ then $V \in{ }_{B} \mathcal{M}$ by

$$
\left(x \# e_{g}\right) v=\beta(g,|v|) x v, \quad x \in B, \quad g \in G, \quad v \in V .
$$

The reciprocal holds provided that $\beta$ is non degenerate. Indeed, if $\xi \in A$, then there exists a unique $h_{\xi} \in G$ such that $\xi(g)=\beta\left(g, h_{\xi}\right)$, for all $g \in G$. Let $V \in B_{G} \mathcal{M}$. Since $G$ acts in $V, V=\oplus_{\xi \in A} V_{\xi}$. Hence, $V$ is $G$-graded with $V_{h_{\xi}}=V_{\xi}$. The action of $B$ is the restriction of the action of $B_{G}$, so $V \in{ }_{B} \mathcal{G \mathcal { M }}$. In this way, the abelian categories ${ }_{B} \mathcal{G \mathcal { M }}$ and ${ }_{B_{G}} \mathcal{M}$ are equivalent.

1.3. Graded coalgebras. A $G$-graded coalgebra is a coalgebra $C$ in $\operatorname{Vect}^{G}$. For instance, the dual coalgebra of a finite dimensional graded algebra is a graded coalgebra. A (left) graded comodule over a graded coalgebra $C$ is a left comodule $V$ such that $V \in \operatorname{Vect}^{G}$ and the coaction $\lambda: V \rightarrow C \otimes V$ is homogeneous. Right graded comodules and graded bicomodules are defined in a similar way. These categories are denoted by ${ }^{C} \mathcal{G M}, \mathcal{G M}^{C}$ and ${ }^{C} \mathcal{G M}{ }^{C}$. Given a graded coalgebra $C$, we denote the coproduct by the following variant of Sweedler's notation: $\Delta(c)=c^{(1)} \otimes c^{(2)}, c \in C$. Analogously, for a graded comodule $V$, we write: $\lambda(v)=v^{(-1)} \otimes v^{(0)}$, for all $v \in V$.

Remark 1.4. Let $C$ be a graded coalgebra and $C_{G}=C \# \mathbb{k} G$ the smash product coalgebra, i. e. the vector space $C \otimes \mathbb{k} G$ with coproduct and counit

$$
\Delta\left(c \# e_{g}\right)=c^{(1)} \# e_{\left|c^{(2)}\right| g} \otimes c^{(2)} \# e_{g}, \quad \varepsilon\left(c \# e_{g}\right)=\varepsilon(c), \quad c \in C, \quad g \in G .
$$

As a consequence, $C_{G}$ is pointed if and only if $C$ is pointed and the grading of the coradical $C_{0}$ is trivial. In this case, we say that $C$ is strictly pointed.

The abelian categories ${ }^{C} \mathcal{G M}$ and ${ }^{C}{ }_{G} \mathcal{M}$ are equivalent. In fact, if $V \in$ ${ }^{C} \mathcal{G M}$, then $V \in C_{G} \mathcal{M}$ by

$$
\lambda_{G}(v)=v^{(-1)} \# e_{\left|v^{(0)}\right|} \otimes v^{(0)}, \quad v \in V .
$$

1.4. Color Hopf algebras. The bicharacter $\beta$ is not needed to define algebras and coalgebras in $\operatorname{Vect}^{G}$, but to define bialgebras and Hopf algebras. First, $\beta$ allows us to define the twisted product between $G$-graded algebras: if $B, B^{\prime}$ are $G$-graded algebras, then $B \otimes B^{\prime}$ is the $G$-graded algebra with multiplication $\left(x \otimes x^{\prime}\right)\left(y \otimes y^{\prime}\right)=\beta\left(\left|x^{\prime}\right|,|y|\right) x y \otimes x^{\prime} y^{\prime}$, for all $x, y \in B, x^{\prime}, y^{\prime} \in B^{\prime}$. A color bialgebra is a bialgebra $B$ in the category $\operatorname{Vect}^{G}$, that is, $B=\oplus_{g \in G} B_{g}$ is a $G$-graded algebra and coalgebra such that $\Delta: B \rightarrow B \otimes B$ and $\varepsilon$ are morphisms of $G$-graded algebras. A color bialgebra $H$ is said a color Hopf 
algebra when the identity map has a convolution inverse $\mathcal{S} \in \operatorname{End}(H)$ (that we shall always assume is bijective.); $\mathcal{S}$ is called the antipode and satisfies

(i) $\mathcal{S}(x y)=\beta(|x|,|y|) \mathcal{S}(y) \mathcal{S}(x)$ and

(ii) $\Delta(\mathcal{S}(x))=\beta\left(\left|x^{(1)}\right|,\left|x^{(2)}\right|\right) \mathcal{S}\left(x^{(2)}\right) \otimes \mathcal{S}\left(x^{(1)}\right)$, for all $x, y \in H$.

Evidently, if $\beta=\varepsilon$ is the trivial bicharacter, then a $(G, \varepsilon)$-color Hopf algebra is a $G$-graded Hopf algebra, that is a Hopf algebra which is $G$-graded algebra and coalgebra with respect to the same grading.

For example, the dual $H^{*}$ of a finite dimensional color Hopf algebra $H$ is again a color Hopf algebra.

Let $H$ be a color Hopf algebra. Then $H$ is a Hopf algebra in $\underset{\mathbb{k} G G}{\mathbb{k} G} y \mathcal{D}$ by Remark 1.2 (iii). Let $H_{G}=H \# \mathbb{k} G$ be the bosonization of $H$, i. e. $H \otimes \mathbb{k} G$ with multiplication and coproduct given by (1.7) and (1.9). Observe that

$$
\mathcal{S}\left(x \# e_{g}\right)=\beta\left(g^{-1}|x|^{-1},|x|\right) \mathcal{S}(x) \# e_{g^{-1}|x|^{-1}}, \quad x \in H, g \in G .
$$

Let ${ }_{H} \mathcal{C M}$ be the category of graded modules with the tensor structure defined as follows. If $V, W \in{ }_{H} \mathrm{e \mathcal {M }}$, then $V \otimes W$ has the underlying tensor product of $G$-graded vector spaces and the action of $H$ given by

$$
h \cdot(v \otimes w)=\beta\left(\left|h^{(2)}\right|,|v|\right) h^{(1)} v \otimes h^{(2)} w, \quad v \in V, \quad w \in W, \quad h \in H .
$$

The functor ${ }_{H} \mathcal{C N} \rightarrow{ }_{H_{G}} \mathcal{M}$ in Remark 1.3 is monoidal. The full subcategory of finite dimensional graded $H$-modules is rigid; if $V$ is such a module, $x \in H_{g}, v^{*} \in V_{h}^{*}=\operatorname{Hom}\left(V_{h^{-1}}, \mathbb{k}\right)$ and $u \in V_{(g h)^{-1}}$, then $H$ acts on $V^{*}$ by

$$
x \cdot v^{*}: V_{(g h)^{-1}} \rightarrow \mathbb{k}, \quad\left(x \cdot v^{*}\right)(u)=\beta(g, h) v^{*}(\mathcal{S}(x) u) .
$$

Similarly, ${ }^{H}$ CM $\mathcal{M}$ denotes the tensor category of graded comodules with the following tensor product. If $V, W \in{ }^{H} \mathrm{C \mathcal {M }}$, then

$$
\lambda(v \otimes w)=\beta\left(\left|v^{(0)}\right|,\left|w^{(-1)}\right|\right) v^{(-1)} w^{(-1)} \otimes v^{(0)} \otimes w^{(0)}, \quad v \in V, \quad w \in W .
$$

The equivalence ${ }^{H} \mathcal{C} \mathcal{M} \simeq{ }_{G} \mathcal{M}$ in Remark 1.4 is monoidal. The subcategory of finite dimensional objects in ${ }^{H} \mathrm{C} \mathcal{M}$ is rigid. Indeed, given such a comodule $V$ and $f \in V^{*}$, the coaction $\lambda(f)=f^{(-1)} \otimes f^{(0)}$ is given by $f^{(-1)} f^{(0)}(v)=$ $\beta\left(\left|v^{(-1)}\right|,\left|v^{(0)}\right|\right) \mathcal{S}^{-1}\left(v^{(-1)}\right) f\left(v^{(0)}\right)$, for $v \in V$.

1.5. Triangular semisimple Hopf algebras and Scheunert's trick. The problem of classifying color Hopf algebras with commutation factor is a particular case of the problem of classifying Hopf algebras in symmetric categories. To start with this general problem, we may consider the categories of finite-dimensional representations of triangular semisimple Hopf algebras. The classification of semisimple triangular Hopf algebras over $\mathbb{k}$ was established in [EG1, EG2], whose notations and definitions we use freely. We next summarize [EG1, Theorem 2.1] and [EG2, Theorem 3.1].

Theorem 1.5. Let $(B, R)$ be a triangular semisimple Hopf algebra over $\mathbb{k}$, with Drinfeld element $u$. Set $R_{u}:=\frac{1}{2}(1 \otimes 1+1 \otimes u+u \otimes 1-u \otimes u)$ and $\widetilde{R}:=R R_{u}$. Then there exist a finite group $L$, a subgroup $K<L$ 
and a minimal twist $J \in \mathbb{k} K \otimes \mathbb{k} K$ such that $(B, \widetilde{R})$ and $\left(\mathbb{k} L^{J}, J_{21}^{-1} J\right)$ are isomorphic as triangular Hopf algebras. Moreover, the data $(L, K, J)$ is unique up to isomorphisms of groups and gauge equivalence of twist.

There is a well-known one-to-one correspondence between bicharacters of $G$ and quasitriangular structures on $\mathbb{k} A$. For, consider the isomorphism of Hopf algebras $\mathbb{k}^{G} \rightarrow \mathbb{k} A$ given by $\delta_{g} \mapsto v_{g}:=\frac{1}{|G|} \sum_{a \in A} a\left(g^{-1}\right) e_{a}$; then the bicharacter $\beta$ corresponds to the R-matrix

$$
R_{\beta}=\sum_{g, h \in G} \beta(g, h) v_{g} \otimes v_{h} .
$$

Under this identification, the Drinfeld element of $\left(\mathbb{k} A, R_{\beta}\right)$ is $u \in \mathbb{k}^{G}$ given by $u(g)=\beta\left(g, g^{-1}\right), g \in G$. Also commutation factors of $G$ correspond to triangular structures on $\mathbb{k} A$. Assume until the end of this Subsection that $\beta$ is a commutation factor of $G$. Then the Drinfeld element $u \in A \simeq G\left(\mathbb{k}^{G}\right)$ is an involution, hence $u(g)=\beta(g, g)$, for $g \in G$, and

$$
\begin{aligned}
\left(R_{\beta}\right)_{u} & =\sum_{g, h \in G} \kappa(g, h) v_{g} \otimes v_{h}, \\
\text { where } & \kappa(g, h)= \begin{cases}1, & \text { if } u(g)=1 \text { or } u(h)=1, \\
-1, & \text { if } u(g)=u(h)=-1 .\end{cases}
\end{aligned}
$$

Consequently the $R$-matrix $\widetilde{R}_{\beta}$ as in Theorem 1.5 has the explicit form

$$
\widetilde{R}_{\beta}=\frac{1}{|G|^{2}} \sum_{g, h \in G} \beta \kappa(g, h) v_{g} \otimes v_{h}
$$

By definition of $\kappa, \beta \kappa: G \times G \rightarrow \mathbb{k}$ is a commutation factor on $G$. Set

$$
K:=(\operatorname{ker} \bar{\chi})^{\perp}=\left\{a \in A:\left.a\right|_{\operatorname{ker} \bar{\chi}}=\epsilon_{A}\right\},
$$

where $\bar{\chi}: G \rightarrow A$ is defined by $\bar{\chi}_{g}(h)=\beta \kappa(g, h), g, h \in G$. $K$ is identified with the group of characters of $G^{\prime}=G / \operatorname{ker} \bar{\chi}$, and $\beta \kappa$ induces a nondegenerate bicharacter $\beta^{\prime}$ on $G^{\prime}$ with $\beta^{\prime}(\bar{g}, \bar{g})=1$ for all $\bar{g} \in G^{\prime}$. Moreover

$$
\widetilde{R}_{\beta}=\sum_{\bar{g}, \bar{h} \in G^{\prime}} \beta^{\prime}(\bar{g}, \bar{h}) v_{\bar{g}} \otimes v_{\bar{h}}, \quad \text { where } v_{\bar{g}}=\frac{1}{\left|G^{\prime}\right|} \sum_{a \in K} a\left(\bar{g}^{-1}\right) e_{a} .
$$

By Scheunert's trick [S], there is a normalized 2-cocycle $\gamma^{\prime}$ on $G^{\prime}$ such that

$$
\beta^{\prime}(\bar{g}, \bar{h})=\frac{\gamma^{\prime}(\bar{g}, \bar{h})}{\gamma^{\prime}(\bar{h}, \bar{g})}, \quad \quad \text { for all } \bar{g}, \bar{h} \in G^{\prime}
$$

A well-known and straightforward calculation shows that

$$
J_{\gamma^{\prime}}=\sum_{\bar{g}, \bar{h} \in G^{\prime}} \gamma^{\prime}(\bar{g}, \bar{h}) v_{\bar{g}} \otimes v_{\bar{h}}
$$

is a minimal twist for $\mathbb{k} K$ such that $\widetilde{R}_{\beta}=\left(J_{\gamma^{\prime}}\right)_{21}^{-1} J_{\gamma^{\prime}}$; but $A$ being abelian, $\mathbb{k} A=\mathbb{k} A^{J_{\gamma^{\prime}}}$ as Hopf algebras. Thus we have: 
Theorem 1.6. The data associated to the triangular semisimple Hopf algebra $\left(\mathbb{k} A, R_{\beta}\right)$ in Theorem 1.5 is $\left(A, K, J_{\gamma^{\prime}}\right)$.

Since $u^{2}=1$, it can be thought of as group morphism $u: G \rightarrow \mathbb{Z}_{2}$. Let $\varsigma$ be the commutation factor on $\mathbb{Z} / 2$ such that Vect ${ }_{\varsigma}^{\mathbb{Z} / 2}$ is the category of supervector spaces; note $\kappa=\varsigma(u \times u)$. Consider the normalized 2-cocycle on $G$ given by $\gamma:=\gamma^{\prime}(p \times p)$, where $p: G \rightarrow G^{\prime}$ is the canonical projection. Then $u$ and $\gamma$ give rise to a braided tensor functor $(F, \phi): \operatorname{Vect}_{\beta}^{G} \rightarrow \operatorname{Vect}_{\varsigma}^{\mathbb{Z} / 2}$ by (1.12), see Remark 1.2 (i). Thus, every color Hopf algebra in Vect ${ }_{\beta}^{G}$ becomes a Hopf superalgebra via $(F, \phi)$.

In fact, the cocycle $\gamma$ induces an equivalence of braided tensor categories $(F, \phi): \operatorname{Vect}_{\beta \kappa}^{G} \rightarrow \operatorname{Vect}_{\varepsilon}^{G}$, where $\varepsilon$ is the trivial bicharacter, see Remark 1.2 (i). (This reflects that $\left(\mathbb{k} A^{J_{\gamma}},\left(J_{\gamma}\right)_{21}^{-1} J_{\gamma}\right)$ is the twist of $(\mathbb{k} A, 1 \otimes 1)$ ). Hence

- If $u=1$, then $(F, \phi): \operatorname{Vect}_{\beta}^{G} \rightarrow \operatorname{Vect}_{\varepsilon}^{G}$ is an equivalence of braided tensor categories, hence any $(G, \beta)$-color Hopf algebra gives rise to a $G$-graded Hopf algebra and vice versa.

- If $u \neq 1$, any $(G, \beta)$-color Hopf algebra gives rise to a $G$-graded Hopf superalgebra and vice versa.

Compare e. g. with $[\mathrm{BaP}$, Section 2]. By these reasons, and taking into account that in Heckenberger's list [H1] there are Nichols algebras that arise neither from usual Lie algebras nor from Lie superalgebras- see the discussion in Ang - we are led to consider bicharacters that are not commutation factors.

\section{CAtegories of modules over color Hopf Algebras}

As always, $G$ is a finite abelian group, $A=\operatorname{Hom}\left(G, \mathbb{k}^{\times}\right), \beta$ is a bicharacter on $G$ and $\chi: G \rightarrow A$ is given by (1.5). We fix a color Hopf algebra $H$. The statements below are basically adaptations of analogous statements for Hopf algebras (and particular cases of statements for braided Hopf algebras in braided tensor categories) and we omit most of the proofs.

2.1. Color Hopf bimodules and Yetter-Drinfeld color modules. A (left) color Hopf module over $H$ is an object $V \in \operatorname{Vect}^{G}$ which is simultaneously a (left) graded module over $H$ and a (left) graded comodule over $H$ such that the coaction $\lambda: V \rightarrow H \otimes V$ is a morphism of graded modules over $H$; here we use the braiding for the action on $H \otimes V$, cf. (1.11). If $U \in \operatorname{Vect}^{G}$, then $H \otimes U$ is a color Hopf module with the regular left action and coaction. If $V$ is a graded comodule over $H$, then $V^{\text {co } H}:=\{v \in V: \lambda(v)=1 \otimes v\}$. The Fundamental theorem of color Hopf modules, a particular case of the analogous theorem in braided tensor categories [T2, says:

Theorem 2.1. If $V$ is a color Hopf module over $H$ then the multiplication $H \otimes V^{\mathrm{co} H} \rightarrow V$ is an isomorphism of color Hopf modules. 
A color Hopf bimodule over $H$ is an object $V \in \operatorname{Vect}^{G}$ which is simultaneously a graded bimodule over $H$ and a graded bicomodule over $H$, and such that the coactions $\lambda: V \rightarrow H \otimes V$ and $\rho: V \rightarrow V \otimes H$ are morphisms of graded bimodules over $H$ (for what we need again the braiding). The category ${ }_{H}^{H} \mathrm{eM}_{H}^{H}$ of color Hopf bimodules over $H$ is tensor one with the tensor product $\otimes_{H}$. A left Yetter-Drinfeld color module over $H$ is an object $V \in \operatorname{Vect}^{G}$ which is simultaneously a left color module over $H$ and a left color comodule over $H$ with compatibility

$$
\lambda(h \cdot v)=\beta\left(\left|h^{(2)}\right|\left|h^{(3)}\right|,\left|v^{(-1)}\right|\right) \beta\left(\left|h^{(2)}\right|,\left|h^{(3)}\right|\right) h^{(1)} v^{(-1)} \mathcal{S}\left(h^{(3)}\right) \otimes h^{(2)} \cdot v^{(0)}
$$

for all $v \in V$ and $h \in H$. As usual, these notions are equivalent.

Proposition 2.2. The category ${ }_{H}^{H}$ yDe of left Yetter-Drinfeld color modules is tensor equivalent to ${ }_{H}^{H} \mathrm{eN}_{H}^{H}$.

Proof. We have functors: ${ }_{H}^{H} \operatorname{e\mathcal {N}}_{H}^{H} \ni M \rightsquigarrow V=M^{\text {co } H} \in \in_{H}^{H}$ yDe, with action $h \cdot v=\beta\left(\left|h^{(2)}\right|, v\right) h^{(1)} v \mathcal{S}\left(h^{(2)}\right)$ and coaction $\lambda$; and ${ }_{H}^{H} y \mathcal{D C} \ni V \rightsquigarrow M=V \otimes H$, with actions and coactions

$$
\begin{array}{rrr}
x \cdot(v \otimes h)=\beta\left(\left|x^{(2)}\right|,|v|\right) x^{(1)} v \otimes x^{(2)} h, & (v \otimes h) \cdot y & =v \otimes h y, \\
\lambda(v \otimes h)=\beta\left(\left|v^{(0)}\right|,\left|h^{(1)}\right|\right) v^{(-1)} h^{(1)} \otimes v^{(0)} \otimes h^{(2)}, & \rho & =\mathrm{id} \otimes \Delta .
\end{array}
$$

These functors are tensor equivalent, inverse of each other.

The tensor category ${ }_{H}^{H} y \mathcal{D C}$ is braided; explicitly, if $V, W \in_{H}^{H} y \mathcal{D C}$, then the braiding $c_{V, W}: V \otimes W \rightarrow W \otimes V$ is

$$
c_{V, W}(v \otimes w)=\beta\left(\left|v^{(0)}\right|,|w|\right) v^{(-1)} w \otimes v^{(0)}, \quad v \in V, \quad w \in W .
$$

Indeed, $c_{V, W}$ is a natural isomorphism in ${ }_{H}^{H} y \mathcal{D C}$ with inverse

$$
c_{V, W}^{-1}(w \otimes v)=\beta\left(\left|v^{(0)}\right|,\left|v^{(-1)} w\right|\right)^{-1} v^{(0)} \otimes \mathcal{S}^{-1}\left(v^{(-1)}\right) w, \quad v \in V, w \in W .
$$

Thus ${ }_{H}^{H} \operatorname{e\mathcal {M}}_{H}^{H}$ is braided, with braiding $c_{M, N}: M \otimes_{H} N \rightarrow N \otimes_{H} M$ given by $m \otimes n \mapsto \beta\left(\left|m^{(0)}\left\|m^{(-1)}|,| n^{(0)}\right\| n^{(1)}\right|\right) m^{(-2)} n^{(0)} \mathcal{S}\left(n^{(1)}\right) \mathcal{S}\left(m^{(-1)}\right) \otimes m^{(0)} n^{(2)}$, for all $m \in M, n \in N$.

Remark 2.3. Let $H$ be a color Hopf algebra and $H_{G}=H \# \mathbb{k} G$. There exists a full embedding of braided tensor categories $i:{ }_{H}^{H} y \mathcal{D C} \hookrightarrow{ }_{H_{G}}^{H_{G}} \mathrm{yD}$; indeed, any $V \in{ }_{H}^{H} y \mathcal{D C}$ becomes a Yetter-Drinfeld module over $H_{G}$ with the structures (1.8) and (1.10).

Example 2.4. Let $H$ be a color Hopf algebra with trivial grading, that is, $H$ is a usual Hopf algebra. If $V \in_{H}^{H} y \mathcal{D}$ and $g \in G$, then $V[g]:=V \in_{H}^{H} y \mathcal{D C}$, where all elements of $V[g]$ have degree $g$. Clearly if $V$ is irreducible in ${ }_{H}^{H} y \mathcal{D}$, then $V[g]$ is irreducible in ${ }_{H}^{H} y \mathcal{D C}$. Reciprocally, if $W=\oplus_{g \in G} W_{g}$ is an irreducible object in ${ }_{H}^{H} y \mathcal{D C}$, then there exists $g \in G$ such that $W=W_{g}$. Hence, $W=U[g]$ with $U:=W$ an irreducible object in ${ }_{H}^{H} y \mathcal{D}$. 
2.2. Bosonization of color Hopf algebras. Since ${ }_{H}^{H} y \mathcal{D C}$ is a braided category, we may consider Hopf algebras in this category. The equivalence between Hopf algebras in ${ }_{H}^{H} y \mathcal{D C}$ and color Hopf algebras with split projection to $H$ goes in the same way as in $[\mathrm{R}, \mathrm{M} 2$. Namely, given a Hopf algebra $R$ in ${ }_{H}^{H} y \mathcal{D C}$, the color Hopf algebra $R \# H$ is $R \otimes H \in \operatorname{Vect}^{G}$ with

$$
\begin{aligned}
(a \# h)(b \# f) & =\beta\left(\left|h^{(2)}\right|,|b|\right) a\left(h^{(1)} \cdot b\right) \# h^{(2)} f, \\
\Delta(a \# h) & =\beta\left(\left|\left(a^{(2)}\right)^{(0)}\right|,\left|h^{(1)}\right|\right) a^{(1)} \#\left(a^{(2)}\right)^{(-1)} h^{(1)} \otimes\left(a^{(2)}\right)^{0} \# h^{(2)}, \\
1_{R \# H} & =1_{R} \# 1_{H}, \quad \varepsilon(a \# h)=\varepsilon_{R}(a) \varepsilon_{H}(h), \\
\mathcal{S}(a \# h) & =\beta\left(\left|a^{(0)}\right|,|h|\right)\left(1_{R} \# \mathcal{S}_{H}\left(a^{(-1)} h\right)\right)\left(\mathcal{S}_{R}\left(a^{(0)}\right) \# 1_{H}\right),
\end{aligned}
$$

for all $a, b \in R$ and $h, f \in H$. By Remark 2.3, $\mathfrak{i}(R)$ is a Hopf algebra in ${ }_{H_{G}}^{H_{G}} y \mathcal{D}$. It is straightforward to verify that

$$
(R \# H)_{G} \simeq \mathfrak{i}(R) \# H_{G} .
$$

Conversely, let $\iota: H \hookrightarrow L$ and $\pi: L \rightarrow H$ be morphisms of color Hopf algebras such that $\pi \circ \iota=\mathrm{id}_{H}$. The subalgebra of coinvariants

$$
R:=L^{\operatorname{co} H}=\{x \in L:(\operatorname{id} \otimes \pi) \Delta(x)=x \otimes 1\}
$$

is a Hopf algebra in the category ${ }_{H}^{H} y \mathcal{D C}$ with the following structures

$$
\begin{aligned}
& h . r=\beta\left(\left|h^{(2)}\right|,|r|\right) \iota\left(h^{(1)}\right) r \iota\left(\mathcal{S}\left(h^{(2)}\right)\right), \quad \lambda=(\pi \otimes i d) \Delta, \\
& \Delta_{R}(r)=r^{(1)} \iota \pi \mathcal{S}\left(r^{(2)}\right) \otimes r^{(3)}, \quad \varepsilon_{R}(r)=\varepsilon_{L}(r), \quad \mathcal{S}_{R}(r)=\iota \pi\left(r^{(1)}\right) \mathcal{S}_{L}\left(r^{(2)}\right) .
\end{aligned}
$$

The color Hopf algebras $L$ and $R \# H$ are isomorphic.

Note that $\iota$ and $\pi$ induce morphisms of Hopf algebras $\iota_{G}: H_{G} \hookrightarrow L_{G}$ and $\pi_{G}: L_{G} \rightarrow H_{G}$ such that $\pi_{G} \circ \iota_{G}=\operatorname{id}_{H_{G}}$. Hence $\mathfrak{i}(R)$ coincides with the subalgebra of coinvariants $\left(L_{G}\right)^{\operatorname{co}\left(H_{G}\right)}$, see e. g. [AHS, Lemma 3.1].

2.3. Color Nichols algebras. The structure of a Nichols algebra appeared first in $[\mathrm{N}]$. See $[\mathrm{AS}]$ for a detailed introduction to this construction.

Proposition 2.5. Let $V \in{ }_{H}^{H} y \mathcal{D C}$. Then there is a unique (up to isomorphisms) graded Hopf algebra $\mathfrak{B}(V)=\oplus_{n \in \mathbb{N}_{0}} \mathfrak{B}^{n}(V)$ in ${ }_{H}^{H} y \mathcal{D C}$ such that:

(i) $\mathfrak{B}^{0}(V) \simeq \mathbb{k}$,

(ii) $V \simeq \mathfrak{B}^{1}(V)=\mathcal{P}(\mathfrak{B}(V))$ (the space of primitive elements),

(iii) $\mathfrak{B}^{1}(V)$ generates the algebra $\mathfrak{B}(V)$.

We have a similar explicit description of $\mathfrak{B}(V)$ as in [AAY, 1.7]. Then, by Remark 2.3. it follows that

$$
\mathfrak{B}(\mathfrak{i}(V))=\mathfrak{i}(\mathfrak{B}(V)) .
$$


2.4. The lifting method for color Hopf algebras. Let $H$ be a color Hopf algebra. The coradical $H_{0}$ of $H$ is a graded subcoalgebra of $H$. Assume that $H_{0}$ is also a subalgebra of $H$. Because of this assumption on $H$, the coradical filtration $\left\{H_{n}\right\}$ is also a filtration of algebras and the associated graded algebra gr $H$ is a color Hopf algebra. Since the homogeneous projection $\pi:$ gr $H \longrightarrow H_{0}$ is a morphism of color Hopf algebras that splits the inclusion map, the algebra of left coinvariants $R=(\operatorname{gr} H)^{\mathrm{co} \pi}$ is a braided Hopf algebra in ${ }_{H_{0}}^{H_{0}} y \mathcal{D}$ and $\operatorname{gr} H \simeq R \# H_{0} ; R=\oplus_{n \geq 0} R(n)$ and $R(0)=\mathbb{k} 1, R(1)=\mathcal{P}(R)$. Consequently the subalgebra generated by $R(1)$ is isomorphic to the Nichols algebra $\mathcal{B}(R(1))$. That is, we can adapt the lifting method AS to the setting of color Hopf algebras. However, the classification problems of color Hopf algebras whose coradical is a subalgebra reduce to classification problems of Hopf algebras with the same property.

Proposition 2.6. Let $H$ be a color Hopf algebra.

(i) The coradical filtration of $H_{G}$ is $\left\{H_{n} \# \mathbb{k} G\right\}$.

(ii) $H_{0}$ is a color Hopf subalgebra of $H$ if and only if $\left(H_{G}\right)_{0}$ is a Hopf subalgebra of $H_{G}$. In this case,

$$
\operatorname{gr}\left(H_{G}\right) \simeq(\operatorname{gr} H)_{G} \simeq\left(R \# H_{0}\right)_{G} \simeq \mathfrak{i}(R) \#\left(H_{0}\right)_{G} .
$$

(iii) The assignement $H \rightsquigarrow H_{G}$ provides a one-to-one correspondence between finite-dimensional strictly pointed color Hopf algebras $H$ and finitedimensional pointed Hopf algebras $\mathcal{H}$ such that:

- $G(\mathcal{H})=G(H) \times G$.

- The inclusion $\mathbb{k} G \hookrightarrow \mathcal{H}$ has a Hopf algebra retraction $\pi: \mathcal{H} \longrightarrow \mathbb{k} G$.

- As Yetter-Drinfeld module, $\mathcal{H}^{\mathrm{co} \pi}$ comes from the grading as in (1.6).

Example 2.7. Let $\Gamma$ be a finite abelian group. Then any finite-dimensional $V$ in ${ }_{\mathbb{k} \Gamma}^{\mathbb{k} \Gamma} y \mathcal{D}$ has a decomposition in direct sum of sub-objects of the form $V_{u}^{\zeta}$, $u \in \Gamma, \zeta \in \widehat{\Gamma}$, where $u$ determines the coaction and $\zeta$ the action. Consider $\mathbb{k} \Gamma$ as a color Hopf algebra with trivial grading so that ${ }_{\mathbb{k} \Gamma}{ }_{\mathrm{k}} \mathrm{y} \mathcal{D} \mathcal{C}$ is a semisimple category. By Example 2.4, any finite-dimensional $V \in \underset{\mathbb{k} \Gamma}{\mathbb{k}} y \mathcal{D} \mathcal{C}$ has a basis $x_{1}, \ldots, x_{\theta}$ such that $x_{j} \in V_{u_{j}}^{\zeta_{j}}\left[t_{j}\right], u_{j} \in \Gamma, \zeta_{j} \in \widehat{\Gamma}$ and $t_{j} \in G$.

Proposition 2.8. Let $u_{j} \in \Gamma, \zeta_{j} \in \widehat{\Gamma}, t_{j} \in G, 1 \leq j \leq \theta$. Set

$$
\widetilde{q}_{i j}=\zeta_{j}\left(u_{i}\right), \quad q_{i j}=\beta\left(t_{i}, t_{j}\right) \widetilde{q}_{i j}, \quad 1 \leq i, j \leq \theta .
$$

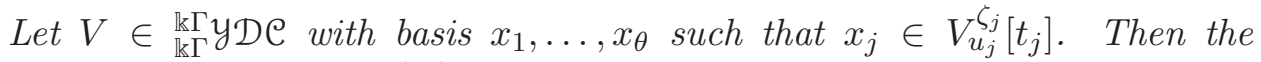
color Nichols algebra $\mathfrak{B}(V)$ has finite dimension if and only if the connected components of the generalized Dynkin diagram corresponding to the matrix $\left(q_{i j}\right)_{1 \leq i, j \leq r}$ belong to the list in [H1].

Proof. It follows directly from (2.1) and (2.3). 


\section{EXAmples Related With QUANTUm Doubles}

In this section we construct examples of color Hopf algebras from Nichols algebras of diagonal type. We start by a general construction of quantum doubles extending that of $\mathrm{H} 2$, where quantum doubles of bosonizations of Nichols algebras by $\mathbb{k} \mathbb{Z}^{\theta}$ are considered. We describe in Subsection 3.1 quantum doubles of realizations over other abelian groups. A similar construction was studied in [Bt] for finite abelian groups; see also [ARS, RaS]. We show how to give colors to these quantum doubles, that can be thought of as generalizations of quantized enveloping (super)algebras. Then we show examples where the color is consistent with the associated Weyl groupoid.

3.1. General construction. We refer to $\mathrm{KS}$, DT for the definition of skew-Hopf pairing between Hopf algebras $B$ and $B^{\prime}$ and the associated Hopf algebra $B \bowtie B^{\prime}$. Let $\mathbb{I}=\{1, \ldots, \theta\}$. We consider a datum

$$
\mathcal{E}=\left(\mathbf{q}, \Gamma, \Lambda,\left(g_{i}\right)_{i \in \mathbb{I}},\left(\gamma_{i}\right)_{i \in \mathbb{I}},\left(h_{i}\right)_{i \in \mathbb{I}},\left(\lambda_{i}\right)_{i \in \mathbb{I}}, \bar{\mu}\right),
$$

where

- $\mathbf{q}=\left(q_{i j}\right)_{i, j \in \mathbb{I}}$ has entries in $\mathbb{k}^{\times}$,

- $\Gamma, \Lambda$ are two abelian groups,

- $g_{i} \in \Gamma, \gamma_{j} \in \widehat{\Gamma}$ are such that $\gamma_{j}\left(g_{i}\right)=q_{i j}$,

- $h_{i} \in \Lambda, \lambda_{j} \in \widehat{\Lambda}$ are such that $\lambda_{j}\left(h_{i}\right)=q_{j i}$,

- $\bar{\mu}: \Gamma \times \Lambda \rightarrow \mathbb{k}^{\times}$is a $\mathbb{Z}$-bilinear form such that $\bar{\mu}\left(g_{i}, h_{j}\right)=q_{i j}$.

Let $\bar{\mu}: \mathbb{k} \Gamma \otimes \mathbb{k} \Lambda \rightarrow \mathbb{k}$ be the skew-Hopf pairing associated to $\bar{\mu}$.

For example, [H2, Section 4] deals with the case $\Gamma=\Lambda=\mathbb{Z}^{\theta}$, while we are interested in the case $\Gamma=\mathbb{Z}^{\theta} \times G, \Lambda=\mathbb{Z}^{\theta} \times A, G$ our finite abelian group. Another interesting example is: $\Gamma$ a finite abelian group, $\Lambda=\widehat{\Gamma}$, where $h_{i}=\gamma_{i}, \lambda_{i}=g_{i}$ under the canonical identification of $\Gamma$ with the group of characters of $\widehat{\Gamma}$, and $\bar{\mu}$ is the evaluation.

First we attach to the datum $\mathcal{E}$ two Yetter-Drinfeld modules

$$
\begin{aligned}
& V \in \in_{\mathbb{k} \Gamma}^{\mathbb{k} \Gamma} y \mathcal{D} \quad \text { with a fixed basis } \quad E_{1}, \ldots, E_{\theta}, \quad E_{i} \in V_{g_{i}}^{\gamma_{i}}, \\
& W \in_{\mathbb{k} \Lambda}^{\mathbb{k} \Lambda} y \mathcal{D} \quad \text { with a fixed basis } \quad F_{1}, \ldots, F_{\theta}, \quad F_{i} \in W_{h_{i}}^{\lambda_{i}} \text {. }
\end{aligned}
$$

Following [H2], we consider the Hopf algebras

- $\mathcal{A}=T(V) \# \mathbb{k} \Gamma$, i. e. the algebra over $\mathbb{k} \Gamma$ generated by elements $E_{i}$, $i \in \mathbb{I}$, with relations $g E_{i}=\gamma_{i}(g) E_{i} g$, and coproduct determined by:

$\Delta(g)=g \otimes g, \quad g \in \Gamma, \quad \Delta\left(E_{i}\right)=E_{i} \otimes 1+g_{i} \otimes E_{i}, \quad i \in \mathbb{I}$.

- $\mathcal{A}^{\prime}=(T(W) \# \mathbb{k} \Lambda)^{\text {cop }}$, the algebra over $\mathbb{k} \Lambda$ generated by elements $F_{i}$, $i \in \mathbb{I}$, and relations $h F_{i}=\lambda_{i}(h) F_{i} h$. The coproduct is given by:

$$
\Delta(h)=h \otimes h, \quad h \in \Lambda, \quad \Delta\left(F_{i}\right)=F_{i} \otimes h_{i}+1 \otimes F_{i}, \quad i \in \mathbb{I} .
$$


Proposition 3.1. There exists a unique skew-Hopf pairing $\mu: \mathcal{A} \otimes \mathcal{A}^{\prime} \rightarrow \mathbb{k}$ such that, for all $i, j \in \mathbb{I}, g \in \Gamma, h \in \Lambda, E \in T(V)$ and $F \in T(W)$,

$$
\begin{aligned}
\left.\mu\right|_{\mathbb{k} \Gamma \otimes k \Lambda}=\bar{\mu}, \quad \mu\left(E_{i}, F_{j}\right) & =-\delta_{i, j}, \quad \mu\left(E_{i}, h\right)=\mu\left(g, F_{i}\right)=0, \\
\mu(E g, F h) & =\mu(E, F) \mu(g, h) .
\end{aligned}
$$

Proof. Analogous to that of [H2, Proposition 4.3]. In fact we can define an algebra homomorphism $\Upsilon: \mathcal{A} \rightarrow\left(\mathcal{A}^{\prime}\right)^{*}$, by sending $g \mapsto \bar{g}, E_{i} \mapsto \overline{E_{i}}$, the linear maps determined by the following equations

$$
\bar{g}(F h)=\epsilon(F) \bar{\mu}(g, h), \quad \overline{E_{i}}\left(F_{j_{1}} \cdots F_{j_{n}} h\right)=-\delta_{n, 1} \delta_{j_{1}, i} .
$$

The map $\Upsilon$ is well-defined; indeed, $\bar{g} \overline{g^{\prime}}=\overline{g g^{\prime}}, \bar{g} \overline{E_{i}}=\gamma_{i}(g) \overline{E_{i}} \bar{g}$, for all $g, g^{\prime} \in$ $\Gamma, 1 \leq i \leq \theta$. Therefore the bilinear form satisfying (3.3) and (3.4) is defined by $\mu(a, b):=\Upsilon(a)(b)$. The rest of the proof follows as in loc. cit.

Let $\mathcal{B}=\mathcal{B}(V) \# \mathbb{k} \Gamma$ and $\mathcal{B}^{\prime}=(\mathcal{B}(W) \# \mathbb{k} \Lambda)^{\text {cop }}$. There exist canonical surjective Hopf algebra maps $\pi_{V}: \mathcal{A} \rightarrow \mathcal{B}, \pi_{W}: \mathcal{A}^{\prime} \rightarrow \mathcal{B}^{\prime}$. If $\mathcal{J}(V), \mathcal{J}(W)$ denote the ideals of $T(V), T(W)$ defining the corresponding Nichols algebras, then

$$
\operatorname{ker} \pi_{V}=\mathcal{J}(V) \Gamma, \quad \operatorname{ker} \pi_{W}=\mathcal{J}(W) \Lambda .
$$

We prove now [H2, Theorem 5.8] in our general setting; cf. also [ARS, Bt.

Theorem 3.2. The skew-Hopf pairing $\mu$ of Proposition 3.1 induces a skewHopf pairing $\mu: \mathcal{B} \otimes \mathcal{B}^{\prime} \rightarrow \mathbb{k}$, whose restriction to $\mathcal{B}(V) \otimes \mathcal{B}(W)$ is nondegenerate; $\mu$ is non-degenerate if and only if $\bar{\mu}$ is non-degenerate.

Proof. Set $\mathcal{V}^{+}=T(V) \# \mathbb{Z}^{\theta}$ (and call $\left(K_{i}\right)_{i \in \mathbb{I}}$ the canonical basis of this $\mathbb{Z}^{\theta}$ ); $\mathcal{V}^{-}=T(W) \# \mathbb{Z}^{\theta}$ (and call $\left(L_{i}\right)_{i \in \mathbb{I}}$ the canonical basis of this second copy of $\left.\mathbb{Z}^{\theta}\right), \nu: \mathcal{V}^{+} \otimes \mathcal{V}^{-} \rightarrow \mathbb{k}$ the bilinear form as in Proposition 3.1 but with respect with these copies of $\mathbb{Z}^{\theta}$, cf. [H2, Proposition 4.3]. There exist Hopf algebra maps $\alpha_{V}: \mathcal{V}^{+} \rightarrow \mathcal{A}, \alpha_{W}: \mathcal{V}^{-} \rightarrow \mathcal{A}^{\prime}$, determined by the conditions $\alpha_{V}\left(K_{i}\right)=g_{i},\left.\alpha_{V}\right|_{T(V)}=\operatorname{id}_{T(V)}, \alpha_{W}\left(L_{i}\right)=h_{i},\left.\alpha_{W}\right|_{T(W)}=\operatorname{id}_{T(W)}, i \in \mathbb{I}$. Then

$$
\nu(x, y)=\mu\left(\alpha_{V}(x), \alpha_{W}(y)\right), \quad \text { for all } x \in \mathcal{V}^{+}, y \in \mathcal{V}_{-} .
$$

We claim that $\mu\left(\mathcal{J}(V) \Gamma, \mathcal{A}^{\prime}\right)=0, \mu(\mathcal{A}, \mathcal{J}(W) \Lambda)=0$. To prove this, we use (3.4) and argue as in the proof of [H2, Theorem 5.8]. Therefore we induce a bilinear form $\mu: \mathcal{B} \otimes \mathcal{B}^{\prime} \rightarrow \mathbb{k}$, which is a skew-Hopf pairing because $\pi_{V}, \pi_{W}$ are Hopf algebra morphisms. The restriction of the pairing to $\mathcal{B}(V) \otimes \mathcal{B}(W)$ is non-degenerate because of (3.5) and again the proof of [H2, Theorem 5.8]. The last claim follows from the preceding and (3.4).

Definition 3.3. Let $D(\varepsilon)$ be the Hopf algebra obtained from $\mathcal{B} \otimes \mathcal{B}^{\prime}$ twisting by the cocycle associated to $\mu[\mathrm{DT}, \mathrm{KS}$. It is presented by generators $g \in \Gamma$, $h \in \Lambda, E_{i}, F_{i}, i \in \mathbb{I}$, the relations in $\mathcal{J}(V), \mathcal{J}(W)$, those of $\Gamma \times \Lambda$, and

$$
\begin{aligned}
g E_{i} & =\gamma_{i}(g) E_{i} g, & h E_{i} & =\mu\left(g_{i}, h\right)^{-1} E_{i} h, \\
h F_{i} & =\lambda_{i}(h) F_{i} h, & g F_{i} & =\mu\left(g, h_{i}\right)^{-1} F_{i} g, \\
E_{i} F_{j}-F_{j} E_{i} & =\delta_{i j}\left(g_{i}-h_{i}\right), & i, j & \in \mathbb{I}, g \in \Gamma, h \in \Lambda .
\end{aligned}
$$


3.2. Coloring quantum doubles. We now define a color version of the Hopf algebra $D(\mathcal{E})$ above, for the pair $(G, \beta)$; we assume that $\beta$ is nondegenerate in this Subsection. Hence the maps $\chi, \chi^{o}: G \rightarrow A$ as in (1.5) are isomorphisms of groups. To start with, we consider the following datum:

- A matrix $\mathbf{q}=\left(q_{i j}\right)_{i, j \in \mathbb{I}} \in\left(\mathbb{k}^{\times}\right)^{\mathbb{I} \times \mathbb{I}}$, such that $q_{i i} \neq 1$, for all $i \in \mathbb{I}$.

- $t_{i} \in G$ for all $i \in \mathbb{I}$.

- $\Gamma=\Gamma_{0} \times G, \Gamma_{0}$ free abelian with basis $K_{1}, \ldots, K_{\theta}$.

- $\Lambda=\Lambda_{0} \times A, \Lambda_{0}$ free abelian with basis $L_{1}, \ldots, L_{\theta}$.

Set $\xi_{i}=\chi_{t_{i}} \in A, \widetilde{q}_{i j}=\beta\left(t_{i}, t_{j}\right)^{-1} q_{i j}=\xi_{j}\left(t_{i}\right)^{-1} q_{i j}, i, j \in \mathbb{I}$. Let $\mathcal{E}=$ $\left(\mathbf{q}, \Gamma, \Lambda,\left(g_{i}\right)_{i \in \mathbb{I}},\left(\gamma_{i}\right)_{i \in \mathbb{I}},\left(h_{i}\right)_{i \in \mathbb{I}},\left(\lambda_{i}\right)_{i \in \mathbb{I}}, \bar{\mu}\right)$, where

$\circ g_{i}=\left(K_{i}, t_{i}\right) \in \Gamma$.

$\circ \gamma_{j}=\left(\zeta_{j}, \xi_{j}\right) \in \widehat{\Gamma}$, where $\zeta_{j} \in \widehat{\Gamma_{0}}$ is given by $\zeta_{j}\left(K_{i}\right)=\widetilde{q}_{i j}, j \in \mathbb{I}$.

$\circ h_{i}=\left(L_{i}, \xi_{i}\right) \in \Lambda$.

○ $\lambda_{j}=\left(\eta_{j}, t_{j}\right) \in \widehat{\Lambda}$ where $\eta_{j} \in \widehat{\Lambda_{0}}$ is given by $\eta_{j}\left(L_{i}\right)=\widetilde{q}_{j i}, i \in \mathbb{I}$.

$\circ \bar{\mu}: \Gamma \times \Lambda \rightarrow \mathbb{k}^{\times}$is given by $\bar{\mu}\left(\left(K_{i}, t\right),\left(L_{j}, \alpha\right)\right)=\widetilde{q}_{i j} \alpha(t)$.

We shall say that $\mathcal{E}$ is a datum, or a datum for $\mathbf{q}$ if emphasis is needed.

Let $D(\varepsilon)$ be the Hopf algebra as in Definition 3.3 , presented by generators $E_{i}, F_{i}, K_{i}^{ \pm 1}, L_{i}^{ \pm 1}, i \in \mathbb{I}, t \in G, \xi \in A$ with the relations defining the Nichols algebras generated by $E_{i}$ and $F_{i}, i \in \mathbb{I}$; those of $\Gamma \times \Lambda$, and

$$
\begin{array}{ll}
K_{i} E_{j} K_{i}^{-1}=\widetilde{q}_{i j} E_{j}, & K_{i} F_{j} K_{i}^{-1}=\widetilde{q}_{i j}^{-1} F_{j}, \\
L_{i} E_{j} L_{i}^{-1}=\widetilde{q}_{j i}^{-1} E_{j}, & L_{i} F_{j} L_{i}^{-1}=\widetilde{q}_{j i} F_{j}, \\
t E_{j} t^{-1}=\xi_{j}(t) E_{j}=\beta\left(t, t_{j}\right) E_{j}, & t F_{j} t^{-1}=\xi_{j}(t)^{-1} F_{j}, \\
\xi E_{j} \xi^{-1}=\xi\left(t_{j}\right)^{-1} E_{j}, & \xi F_{j} \xi^{-1}=\xi\left(t_{j}\right) F_{j}, \\
E_{i} F_{j}-F_{j} E_{i}=\delta_{i j}\left(t_{i} K_{i}-\xi_{i} L_{i}\right), & i, j \in \mathbb{I}, t \in G, \xi \in A .
\end{array}
$$

Here the elements of $\Gamma \times \Lambda$ are group-like and

$$
\Delta\left(E_{i}\right)=E_{i} \otimes 1+K_{i} t_{i} \otimes E_{i}, \quad \Delta\left(F_{i}\right)=F_{i} \otimes L_{i} \xi_{i}+1 \otimes F_{i} .
$$

Given $t \in G, t \chi_{t}^{o}$ is a central element of $D(\varepsilon)$. Let $I(\varepsilon)$ be the Hopf ideal of $U(\mathcal{E})$ generated by $\left\{t \chi_{t}^{o}-1, t \in G\right\}$ and $U(\mathcal{E}):=D(\mathcal{E}) / I(\mathcal{E})$ the corresponding quotient Hopf algebra; it has a presentation by generators $E_{i}$, $F_{i}, K_{i}^{ \pm 1}, L_{i}^{ \pm 1}, i \in \mathbb{I}, t \in G$ and analogous relations. Our goal is to obtain color Hopf algebras from a factorization $U(\mathcal{E}) \simeq R(\mathcal{E}) \# \mathbb{k} G$. Therefore we analyze first such possible factorizations.

Proposition 3.4. There exists a one-to-one correspondence between

(i) group homomorphisms $\bar{\pi}: \Gamma_{0} \rightarrow G$ and

(ii) Hopf algebra retractions $\pi: U(\mathcal{E}) \longrightarrow \mathbb{k} G$ of $\mathbb{k} G \hookrightarrow U(\mathcal{E})$.

Indeed, given $\bar{\pi}: \Gamma_{0} \rightarrow G$, we define $\pi: U(\mathcal{E}) \rightarrow \mathbb{k} G$ by $\pi(t)=t, t \in G$, and $\pi\left(K_{i}\right)=\bar{\pi}\left(K_{i}\right), \pi\left(L_{i}\right)=t_{i} \bar{\pi}\left(K_{i}\right) \chi^{-1}\left(\xi_{i}\right), \pi\left(E_{i}\right)=\pi\left(F_{i}\right)=0, i \in \mathbb{I}$. 
Proof. It is easy to see that the extension of $\bar{\pi}: \Gamma_{0} \rightarrow \mathbb{k} G$ to $\pi: U(\mathcal{E}) \rightarrow$ $\mathbb{k} G$ as above is a Hopf algebra map. Conversely, let $\pi: U(\mathcal{E}) \rightarrow \mathbb{k} G$ be a Hopf algebra retraction of $\mathbb{k} G \hookrightarrow U(\mathcal{E})$. Since $\pi\left(K_{i}\right), \pi\left(L_{i}\right)$ are grouplikes, $\left.\pi\right|_{\Gamma_{0} \times \Lambda_{0}}$ is a group homomorphism. By (3.6), (3.8) and the hypothesis $q_{i i} \neq 1, \pi\left(E_{i}\right)=0=\pi\left(F_{i}\right)$. Hence $\pi\left(L_{i}\right)=t_{i} \bar{\pi}\left(K_{i}\right) \chi^{-1}\left(\xi_{i}\right)$ by (3.10).

Let $\pi: U(\varepsilon) \rightarrow \mathbb{k} G$ be a Hopf algebra map as in Proposition 3.4 and $R(\varepsilon)=U(\varepsilon)^{\text {co } \pi}$, a braided Hopf algebra in $\mathbb{R} G G_{\mathbb{K} G} \mathcal{D}$. Notice that $R(\varepsilon)$ is the subalgebra of $U(\mathcal{E})$ generated by $E_{i}, \widetilde{K}_{i}=K_{i} \pi\left(K_{i}\right)^{-1}, \widetilde{L}_{i}=L_{i} \pi\left(L_{i}\right)^{-1}$, $\widetilde{F}_{i}=F_{i} t_{i}^{-1} \pi\left(K_{i}\right)^{-1}, i \in \mathbb{I}$. Indeed the later is contained in the former; but the $\mathbb{Z}^{\theta}$-homogeneous components coincide, from $U(\mathcal{E})=R(\mathcal{E}) \# \mathbb{k} G$ and $U(\mathcal{E}) \simeq U^{+}(\chi, \beta) \otimes\left(\mathbb{k} \mathbb{Z}^{2 \theta} \otimes \mathbb{k} G\right) \otimes U^{-}(\chi, \beta)$.

Next we decide when the braided Hopf algebra $R(\mathcal{E})$ is color.

Proposition 3.5. $R(\mathcal{E})$ is a color Hopf algebra if and only if $\bar{\pi}$ is trivial.

Proof. Since $\lambda=(\pi \otimes \mathrm{id}) \Delta$ is the coproduct in $R(\mathcal{E}) \# \mathbb{k} G, \lambda\left(\widetilde{K}_{i}\right)=1 \otimes \widetilde{K}_{i}$, $\lambda\left(\widetilde{L}_{i}\right)=1 \otimes \widetilde{L}_{i}, \lambda\left(E_{i}\right)=\bar{\pi}\left(K_{i}\right) t_{i} \otimes E_{i}$ and $\lambda\left(\widetilde{F}_{i}\right)=t_{i}^{-1} \bar{\pi}\left(K_{i}\right)^{-1} \otimes \widetilde{F}_{i}$. We have to check that the action is given by (1.6). But $t \cdot E_{i}=\beta\left(t, t_{i}\right) E_{i}$ and $t \cdot \widetilde{F}_{i}=\beta\left(t, t_{i}^{-1}\right) \widetilde{F}_{i}, t \in \Gamma$. Since $\beta$ is non-degenerate, $R(\mathcal{E})$ is a color Hopf algebra if and only if $\bar{\pi}\left(K_{i}\right)=1$.

Proposition 3.5 gives many examples of color Hopf algebras, namely $R(\mathcal{E})$, by taking $\bar{\pi}$ trivial.

An important invariant of Nichols algebras of diagonal type is the Weyl groupoid, see [H1 for more details and [AHS for a generalization. Let $\mathbf{q}=\left(q_{i j}\right)_{i, j \in \mathbb{I}} \in\left(\mathbb{k}^{\times}\right)^{\mathbb{I} \times \mathbb{I}}$, such that $q_{i i} \neq 1$, for all $i \in \mathbb{I}$, as above. Define $a_{p p}=2$ and for $j \neq p \in \mathbb{I}$

$$
a_{p j}:=-\min \left\{n:\left(1+q_{p p}+\cdots+q_{p p}^{n}\right)\left(1-q_{p p}^{n} q_{p j} q_{j p}\right)=0\right\} .
$$

Let $p \in \mathbb{I}$ such that all the $a_{p j}$ 's are finite, $j \in \mathbb{I}$. We define

- reflections $s_{p}: \Gamma_{0} \rightarrow \Gamma_{0}$ and $s_{p}: \Lambda_{0} \rightarrow \Lambda_{0}$ by

$$
s_{p}\left(K_{j}\right)=K_{j} K_{p}^{-a_{p j}}, \quad s_{p}\left(L_{j}\right)=L_{j} L_{p}^{-a_{p j}}, \quad i \in \mathbb{I},
$$

- the $p$-reflected matrix $s_{p}^{*} \mathbf{q}=\left(q_{i j}^{\prime}\right)$, where

$$
q_{i j}^{\prime}=q_{i j} q_{p j}^{-a_{p i}} q_{i p}^{-a_{p j}} q_{p p}^{a_{p i} a_{p j}}, \quad i, j \in \mathbb{I} .
$$

The Weyl equivalence relation on the matrices $\mathbf{q}$ as above is induced by $\mathbf{q} \sim s_{p}^{*} \mathbf{q}$, for each $p$ such that all the $a_{p j}$ 's are finite. Indeed, $s_{p}^{*}\left(s_{p}^{*} \mathbf{q}\right)=\mathbf{q}$ [H2]. We now extend the Weyl equivalence to data $\mathcal{E}$ as above by the following rule. Let $p \in \mathbb{I}$ such that all the $a_{p j}$ 's are finite, $j \in \mathbb{I}$. Let

$$
s_{p}^{*} \mathcal{E}=\mathcal{E}^{\prime}=\left(\mathbf{q}^{\prime}, \Gamma, \Lambda,\left(g_{i}^{\prime}\right)_{i \in \mathbb{I}},\left(\gamma_{i}^{\prime}\right)_{i \in \mathbb{I}},\left(h_{i}^{\prime}\right)_{i \in \mathbb{I}},\left(\lambda_{i}^{\prime}\right)_{i \in \mathbb{I}}, \bar{\mu}\right),
$$

be the datum given by 
- $\mathbf{q}^{\prime}=s_{p}^{*} \mathbf{q}$

- $g_{i}^{\prime}=g_{i} g_{p}^{-a_{p i}}$; thus the basis of $\Gamma_{0}$ for $\mathcal{E}^{\prime}$ is $K_{1}^{\prime}=s_{p}\left(K_{1}\right), \ldots, K_{\theta}^{\prime}=$ $s_{p}\left(K_{\theta}\right)$, while $t_{i}^{\prime}=t_{i} t_{p}^{-a_{p i}}$. It follows that $\widetilde{q}_{i j}^{\prime}=\beta\left(t_{i}^{\prime}, t_{j}^{\prime}\right)^{-1} q_{i j}^{\prime}$, satisfies

$$
\widetilde{q}_{i j}^{\prime}=\widetilde{q}_{i j}\left(\widetilde{q}_{p j}\right)^{-a_{p i}}\left(\widetilde{q}_{i p}\right)^{-a_{p j}}\left(\widetilde{q}_{p p}\right)^{a_{p i} a_{p j}}, \quad i, j \in \mathbb{I} .
$$

- $\gamma_{j}^{\prime}=\left(\zeta_{j}^{\prime}, \xi_{j}^{\prime}\right) \in \widehat{\Gamma}$, where $\xi_{j}^{\prime}=\chi_{t_{j}^{\prime}}$ and $\zeta_{j}^{\prime} \in \widehat{\Gamma_{0}}$ is given by $\zeta_{j}^{\prime}\left(K_{i}^{\prime}\right)=\widetilde{q}_{i j}^{\prime}$, $j \in \mathbb{I}$. Hence $\xi_{j}^{\prime}=\xi_{j} \xi_{p}^{-a_{p j}}, \zeta_{j}^{\prime}=\zeta_{j} \zeta_{p}^{-a_{p j}}$ and then $\gamma_{j}^{\prime}=\gamma_{j} \gamma_{p}^{-a_{p j}}$.

- $h_{i}^{\prime}=h_{i} h_{p}^{-a_{p i}}$; thus the basis of $\Lambda_{0}$ for $\mathcal{E}^{\prime}$ is $L_{1}^{\prime}=s_{p}\left(L_{1}\right), \ldots, L_{\theta}^{\prime}=$ $s_{p}\left(L_{\theta}\right)$.

- $\lambda_{j}^{\prime}=\left(\eta_{j}^{\prime}, t_{j}^{\prime}\right) \in \widehat{\Lambda}$ where $\eta_{j}^{\prime} \in \widehat{\Lambda_{0}}$ is given by $\eta_{j}^{\prime}\left(L_{i}^{\prime}\right)=\widetilde{q}_{j i}^{\prime}, i \in \mathbb{I}$. Hence $\eta_{j}^{\prime}=\eta_{j} \eta_{p}^{-a_{p j}}$ and $\lambda_{j}^{\prime}=\lambda_{j} \lambda_{p}^{-a_{p j}}$.

Notice that the bilinear form $\bar{\mu}$ from $\mathcal{E}$ satisfies $\bar{\mu}\left(\left(K_{i}^{\prime}, t\right),\left(L_{j}^{\prime}, \alpha\right)\right)=\widetilde{q}_{i j}^{\prime} \alpha(t)$.

Definition 3.6. Let $\left(\mathbf{q}^{(b)}\right)_{b \in B}$ be the Weyl equivalence class of $\mathbf{q}$. A family of data $\mathcal{E}^{(b)}$ for $\mathbf{q}^{(b)}$, for $b \in B$, is consistently colored when $\mathcal{E}^{(d)}=s_{p}^{*} \mathcal{E}^{(b)}$ for every two adjacent $b, d \in B$ (that is $\mathbf{q}^{(d)}=s_{p}^{*} \mathbf{q}^{(b)}$ for some $p$ ).

The construction above shows that a datum $\mathcal{E}$ for $\mathbf{q}$ determines a consistently colored family of data $\mathcal{E}^{(b)}$ for the Weyl equivalence class of $\mathbf{q}$.

Recall from [H1] that the generalized Dynkin diagram of $\mathbf{q}$ has set of vertices $\mathbb{I}$, each vertex labeled with $q_{i i}$, and arrows between $i, j$ only when $q_{i j} q_{j i} \neq 1$, labeled with this scalar.

We introduce also color Dynkin diagrams for the corresponding matrices $\widetilde{\mathbf{q}}$. It is a diagram with vertices in $\mathbb{I}$, where each vertex $i$ is labeled with the degree $t_{i}$ on $G$ and the scalar $\widetilde{q}_{i i}$. Now we put the following set of arrows:

- an arrow between $i, j \in \mathbb{I}$ when $\widetilde{q}_{i j} \widetilde{q}_{j i} \neq 1$, labeled with this scalar;

- an arrow between $i, j \in \mathbb{I}$ when $\widetilde{q}_{i j} \widetilde{q}_{j i}=1$ but $\beta\left(t_{i}, t_{j}\right) \beta\left(t_{j}, t_{i}\right) \neq 1$, with no labels;

- no arrow between $i, j \in \mathbb{I}$ when $\widetilde{q}_{i j} \widetilde{q}_{j i}=\beta\left(t_{i}, t_{j}\right) \beta\left(t_{j}, t_{i}\right)=1$; in particular, when $\widetilde{q}_{i j} \widetilde{q}_{j i}=1$, and either $t_{i}=e$ or $t_{j}=e$.

We show now examples of consistently colored families of data.

Example 3.7. Consider the abelian group $G=C_{2} \times C_{2}=\langle\sigma\rangle \times\langle\nu\rangle$, and the non-degenerate bicharacter $\beta: G \times G \rightarrow \mathbb{k}$ given by:

$$
\beta(\sigma, \sigma)=\beta(\nu, \nu)=\beta(\sigma, \nu)=-1, \quad \beta(\nu, \sigma)=1 .
$$

We fix the following notation for the $G$-grading on colored Dynkin diagrams:

$\circ$ for degree $1, \quad \bullet$ for degree $\sigma, \otimes$ for degree $\nu, \odot$ for degree $\sigma \nu$.

Fix $q \in \mathbb{k}^{\times}, q \neq \pm 1$, and a matrix $\left(q_{i j}\right)_{1 \leq i, j \leq 4}$ associated to the generalized Dynkin diagram of [H1, Table 3, Row 14]:

$$
{ }^{q} \stackrel{q^{-1}}{-} \circ^{-1} \stackrel{-1}{\longrightarrow} \circ^{-1} \stackrel{-q}{\longrightarrow} \circ^{-q^{-1}}
$$


Set $t_{1}=e, t_{2}=t_{4}=\sigma, t_{3}=\nu$. The matrix $\left(\widetilde{q}_{i j}\right)_{1 \leq i, j \leq 4}$ as in Proposition 2.8 has the colored Dynkin diagram

$$
\circ^{q} \stackrel{q^{-1}}{\bullet} \bullet \otimes^{1} \stackrel{q}{q} \bullet^{q^{-1}}
$$

In the following table we present the associated generalized and colored Dynkin diagrams of the associated consistently colored family of data.

\begin{tabular}{|c|c|}
\hline$\left(q_{i j}\right)=\left(\beta\left(t_{i}, t_{j}\right) \widetilde{q}_{i j}\right)$ & $\left(\widetilde{q}_{i j}\right)$ and $G$-grading \\
\hline$\circ_{-1}^{-1}>_{-q^{-1}}^{\circ^{-1}} \circ^{-1} \stackrel{-q}{-1} \circ^{-q^{-1}}$ & $\bullet^{1}$ \\
\hline$\circ^{q} \stackrel{q^{-1}}{\longrightarrow} \circ^{-1} \stackrel{-q}{\longrightarrow} \circ^{-q^{-1}} \stackrel{-q}{\longrightarrow} \circ^{-q^{-1}}$ & $\diamond^{q} \stackrel{q^{-1}}{\longrightarrow} \bullet^{1} \stackrel{q}{\longrightarrow} \otimes^{q^{-1}} \stackrel{q}{\longrightarrow} \bullet^{q^{-1}}$ \\
\hline 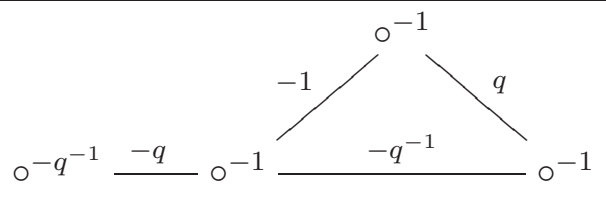 & $\otimes^{q^{-1}} \stackrel{q}{ } \stackrel{q}{1} \odot^{1} \otimes^{1}$ \\
\hline$\circ^{q} \frac{q^{-1}}{\circ^{-1} \overbrace{q}^{-1} \overbrace{}^{-1}-q^{-1}}$ & $\circ^{q} \stackrel{q^{-1}}{ } \odot^{1} \longrightarrow \odot^{1}$ \\
\hline$\circ^{-1} \overbrace{q}^{-1} \overbrace{\circ^{-1}}^{-1} \stackrel{q^{-1}}{-1} \circ^{q}$ & 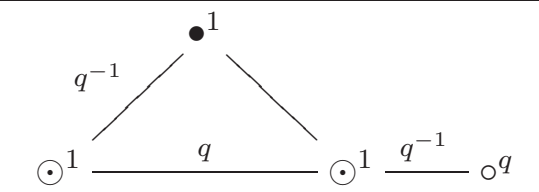 \\
\hline$\circ^{q} \stackrel{q^{-1}}{\longrightarrow} \circ^{q} \stackrel{q^{-1}}{=} \circ^{-1} \stackrel{-q}{\longrightarrow} \circ^{-q^{-1}}$ & $\circ^{q} \stackrel{q^{-1}}{\longrightarrow} \circ^{q} \stackrel{q^{-1}}{ } \odot^{1} \stackrel{q}{\longrightarrow} \bullet^{q^{-1}}$ \\
\hline
\end{tabular}

Example 3.8. Let $\sigma$ be a generator of the cyclic group $G=C_{3}$ of order 3 , $\omega$ a primitive third root of unity and $q \in \mathbb{k}^{\times}, q \neq 1, \omega, \omega^{2}$. Let $\left(\widetilde{q}_{i j}\right)_{1 \leq i, j \leq 2}$ be the matrix given by $\widetilde{q}_{i j}=q^{b_{i j}}$, where $b_{11}=0, b_{12}=b_{21}=-1, b_{22}=2$. Let $\beta$ be the bicharacter on $G$ such that $\beta(\sigma, \sigma)=\omega$ and $t_{1}=\sigma, t_{2}=e$. The matrix $\left(q_{i j}=\beta\left(t_{i}, t_{j}\right) \widetilde{q}_{i, j}\right)_{1 \leq i, j \leq 2}$ has the generalized Dynkin diagram

$$
\circ^{\omega} \stackrel{q^{-1}}{{ }^{q}} \text {. }
$$

Its Weyl equivalence class includes only one more diagram, namely

$$
\circ^{\omega} \frac{q \omega^{2}}{{ }^{2} q^{-1} \omega} \text {. }
$$

Then $t_{1}^{\prime}=t_{2}^{\prime}=\sigma^{2}$. If $\circ, \bullet, \otimes$ denote the vertices of degree $1, \sigma, \sigma^{2}$, respectively, the corresponding colored Dynkin diagrams are

$$
\bullet^{1} \stackrel{q^{-1}}{{ }^{q}} \quad \otimes^{1} \stackrel{q}{\longrightarrow} \otimes^{q^{-1}} .
$$


To conclude this subsection, we discuss examples of color Hopf algebras arising from a version of $U(\varepsilon)$ but with only one copy of $\mathbb{Z}^{\theta}$. More precisely, we assume that $\left(\widetilde{q}_{i j}\right)_{1 \leq i, j \leq \theta}$ is symmetric, so $K_{i} L_{i}$ is a central element on $U(\mathcal{E})$, for all $i \in \mathbb{I}$. Fix $\mathcal{U}(\mathcal{E}):=U(\mathcal{E}) / J$, where $J$ is the Hopf ideal of $U(\mathcal{E})$ generated by the set $\left\{K_{i} L_{i}-1: i \in \mathbb{I}\right\}$. The next proposition is an immediate consequence of Propositions 3.4 and 3.5. There is a condition on the elements $t_{i}$, not always satisfied as we will see in Example 3.10 .

Proposition 3.9. Let $\mathcal{U}(\mathcal{E})$ be as above.

(i) There exists a one-to-one correspondence between

(a) group morphisms $\bar{\pi}: \mathbb{Z}^{\theta} \rightarrow G$, such that $\bar{\pi}\left(K_{i}^{2}\right)=t_{i}^{-2}$,

(b) surjective Hopf algebra morphisms $\pi: \mathcal{U}(\mathcal{E}) \rightarrow \mathbb{k} G$ that splits the natural inclusion $\mathbb{k} G \hookrightarrow \mathcal{U}(\mathcal{E})$.

(ii) $\mathcal{U}(\mathcal{E})^{\mathrm{co} \pi}$ is a color Hopf algebra if and only if $t_{i}^{2}=1$, for all $1 \leq i \leq \theta$.

Example 3.10. The next algebra is described in [Y]. It corresponds to our construction in Proposition 3.9 (i) and gives place to a Yetter-Drinfeld Hopf algebra, which is not a color Hopf algebra. Let $\left(\widetilde{q}_{i j}\right)_{1 \leq i, j \leq 2}, \sigma$ and $\omega$ be as in Example 3.8, The algebra $\mathcal{U}(\mathcal{E})$ is presented by generators $E_{i}, F_{i}, K_{i}^{ \pm 1}$, $\sigma, i=1,2$, relations (3.6) , relations from the group and

$$
\begin{aligned}
K_{i} E_{j} & =q^{b_{i j}} E_{j} K_{i}, & K_{i} F_{j} & =q^{-b_{i j}} F_{j} K_{i} \\
\sigma E_{i} & =\omega^{\delta_{1 i}} E_{i} \sigma, & \sigma F_{i} & =\omega^{-\delta_{1 i}} F_{i} \sigma \\
E_{1}^{3} & =\left(\operatorname{ad}_{c} E_{2}\right)^{2} E_{1}=0 & F_{1}^{3} & =\left(\operatorname{ad}_{c} F_{2}\right)^{2} F_{1}=0, \\
E_{i} F_{j}-F_{j} E_{i} & =\delta_{i j}\left(-K_{i} \sigma^{\delta_{1 i}}+K_{i}^{-1} \sigma^{-\delta_{1 i}}\right) . & &
\end{aligned}
$$

Note that $\pi: \mathcal{U}(\mathcal{E}) \rightarrow \mathbb{k} C_{3}$, given by $\pi(\sigma)=\sigma, \pi\left(E_{i}\right)=\pi\left(F_{i}\right)=0$ and $\pi\left(K_{i}\right)=\sigma^{-\delta_{1 i}}$, is a Hopf algebra morphism that splits the natural inclusion $\mathbb{k} C_{3} \hookrightarrow \mathcal{U}(\mathcal{E})$. In our context $t_{1}=\sigma, t_{2}=1$, so $t_{1}^{2} \neq 1$ and $\mathcal{U}(\mathcal{E})^{\operatorname{co} \pi}$ is not a color Hopf algebra by Proposition 3.9 (ii).

\section{Examples of Semisimple color Hopf algebras}

Throughout this section, $G, A, \beta$ and $\chi_{g}$ are as above, see (1.5). If $L<G$ is a subgroup, then $L^{\perp}:=\left\{a \in A:\left.a\right|_{L}=\varepsilon\right\}$. Clearly, if $G_{1}, \ldots, G_{r}$ are subgroups of $G$, then $\left(\cap_{i=1}^{r} G_{i}\right)^{\perp}=\left\langle G_{1}^{\perp} \cup \ldots \cup G_{r}^{\perp}\right\rangle$. We start by a general fact from [Sch, Corollary 2]; see also [Du, formula (9), page 334].

Proposition 4.1. Let $H$ and $P$ be Hopf algebras. Assume that $H$ is an object in ${ }_{P}^{P} y \mathcal{D}$ for some action and coaction, in such a way that it is an algebra and a coalgebra in ${ }_{P}^{P} y \mathcal{D}$. Then the following are equivalent:

(i) $H$ is a Hopf algebra in ${ }_{P}^{P} y \mathcal{D}$.

(ii) The braiding $c: H \otimes H \rightarrow H \otimes H, c(x \otimes y)=x^{(-1)} \cdot y \otimes x^{(0)}, x, y \in H$, is the usual flip. 
Remark 4.2. Let $H$ be a Hopf algebra. Then $H$ is a $G$-graded Hopf algebra if and only if there exists a morphism $\varphi: A \rightarrow$ Aut $H$ (i. e. an action of $A$ on $H$ by Hopf algebra automorphisms); clearly $\operatorname{Stab}_{A}(H)=\langle\sup H\rangle^{\perp}$. Hence $H$ is a $(G, \beta)$-color Hopf algebra if and only if $\left.\beta\right|_{\sup \mathrm{H} \times \sup \mathrm{H}}=1$, if and only if $\chi(\sup H)<\operatorname{Stab}_{A}(H)$.

4.1. Group algebras. In this subsection, we determine when a group algebra $\mathbb{k} \Gamma, \Gamma$ a finite group, is a color Hopf algebra. By Remark 4.2, $\mathbb{k} \Gamma$ is a $G$-graded Hopf algebra if and only if there exists an action by group automorphisms of $A$ on $\Gamma$. Fix such an action; then also $G$ acts on $\Gamma$ via $\chi$. Clearly $\operatorname{Stab}_{A}(\mathbb{k} \Gamma)=\operatorname{Stab}_{A}(\Gamma)$, hence $\beta$ is trivial on $\sup \mathbb{k} \Gamma$ if and only if

$$
\sup \mathbb{k} \Gamma<\chi^{-1}\left(\operatorname{Stab}_{A}(\Gamma)\right)=\operatorname{Stab}_{G}(\Gamma)=\cap_{\eta \in \Gamma} G^{\eta} .
$$

Definition 4.3. A $(G, \beta)$-color group is a group $\Gamma$ provided with an action of $A$ by group automorphisms such that $\left(G^{\eta}\right)^{\perp}<\operatorname{Stab}_{A}(\Gamma)$, for all $\eta \in \Gamma$.

Theorem 4.4. Let $\Gamma$ be a group provided with an action of $A$ by group automorphisms. Then the following are equivalent:

(i) $\mathbb{k} \Gamma$ is a color Hopf algebra,

(ii) $\beta(g, h)=1$, for all $g, h \in \sup \mathbb{k} \Gamma$,

(iii) $\Gamma$ is a $(G, \beta)$-color group,

(iv) $\mathbb{k}^{\Gamma}$ is a color Hopf algebra.

Proof. (i) $\Leftrightarrow$ (ii) follows by from Proposition 4.1. (i) $\Leftrightarrow$ (iv) is clear. (ii) $\Rightarrow$ (iii): Consider $\eta \in \Gamma$ and $a \in\left(G^{\eta}\right)^{\perp}$. Since sup $\mathbb{k} \Gamma \subset G^{\eta}, a \in \operatorname{Stab}_{A}(\Gamma)$. (iii) $\Rightarrow$ (ii): If $g \in \sup \mathbb{k} \Gamma$, then $\chi_{g}(t)=\chi_{t}(g)^{-1}=1$, for all $t \in \chi^{-1}\left(\operatorname{Stab}_{A}(\Gamma)\right)$. Hence, $\chi_{g} \in\left(\cap_{\eta \in \Gamma} G^{\eta}\right)^{\perp}=\left\langle\cup_{\eta \in \Gamma}\left(G^{\eta}\right)^{\perp}\right\rangle \subset \operatorname{Stab}_{A}(\Gamma)$, and consequently, $\beta(g, h)=\chi_{g}(h)=1$, for all $h \in \sup \mathbb{k} \Gamma$.

Example 4.5. Let $G=C_{4}=\langle g\rangle$ be the cyclic group of order $4, \mathbf{i} \in \mathbb{k}$ such that $\mathbf{i}^{2}=-1$ and $\beta: G \times G \rightarrow \mathbb{k}^{\times}, \beta\left(g^{i}, g^{j}\right)=(-1)^{i j}$; then $A=\langle a\rangle$, where $a(g)=\mathbf{i}$. Let $\Gamma=C_{2} \oplus C_{2}=\langle\gamma\rangle \oplus\langle\eta\rangle$ with the action of $A$ given by $a(\gamma)=\gamma$ and $a(\eta)=\gamma+\eta$. Then $\mathbb{k} \Gamma$ is a color Hopf algebra since $\beta(x, y)=1$ for all $x, y \in \sup \mathbb{k} \Gamma=\left\{1, g^{2}\right\}$.

Example 4.6. Let $G=C_{2} \oplus C_{4}=\langle g\rangle \oplus\langle h\rangle, \mathbf{i} \in \mathbb{k}$ such that $\mathbf{i}^{2}=-1$ and $\beta: G \times G \rightarrow \mathbb{k}^{\times}, \beta\left(g^{i}+h^{j}, g^{k}+h^{l}\right)=\mathbf{i}^{i l-j k}$; then $A=\langle a\rangle \oplus\langle b\rangle$, where $a(g)=-1, a(h)=b(g)=1$ and $b(h)=\mathbf{i}$. Let $\Gamma=C_{2} \oplus C_{2}=\langle\gamma\rangle \oplus\langle\eta\rangle$ with the action of $A$ given by $a(\gamma)=b(\gamma)=\gamma$ and $a(\eta)=b(\eta)=\gamma+\eta$. Then $\mathbb{k} \Gamma$ is a color Hopf algebra since $\beta(x, y)=1$ for all $x, y \in \sup \mathbb{k} \Gamma=\left\{1, g+h^{2}\right\}$.

4.2. Abelian extensions in color categories. In this subsection, we analyze color Hopf algebras that arise as abelian extensions.

4.2.1. Abelian extensions. Let $(\Gamma, L)$ be a matched pair of groups [Ma, T1, that is, $L$ and $\Gamma$ are finite groups provided with (right and left) actions $\triangleleft: L \times \Gamma \rightarrow L$ and $\triangleright: L \times \Gamma \rightarrow \Gamma$ satisfying: for all $l, t \in L$ and $\gamma, \eta \in \Gamma$

$$
l \triangleright \gamma \eta=(l \triangleright \gamma)((l \triangleleft \gamma) \triangleright \eta) \text { and } l t \triangleleft \gamma=(l \triangleleft(t \triangleright \gamma))(t \triangleleft \gamma) \text {. }
$$


Let $\sigma: \Gamma \times \Gamma \rightarrow\left(\mathbb{k}^{\times}\right)^{L}$ and $\tau: L \times L \rightarrow\left(\mathbb{k}^{\times}\right)^{\Gamma}$ be normalized 2-cocycles; write $\sigma=\sum_{l \in L} \sigma_{l} \delta_{l}, \tau=\sum_{\gamma \in \Gamma} \tau_{\gamma} \delta_{\gamma}$. We shall always assume that $\sigma$ and $\tau$ satisfy the following normalization conditions: for all $l, t \in L$ and $\gamma, \eta \in \Gamma$

$$
\sigma_{1}(\gamma, \eta)=1, \quad \tau_{1}(l, t)=1
$$

Let $H=\mathbb{k}^{L} \tau_{\sigma_{\sigma}} \mathbb{k} \Gamma$ be the vector space $\mathbb{k}^{L} \otimes \mathbb{k} \Gamma$ with the crossed product algebra and the crossed coproduct coalgebra structures. The multiplication and coproduct of $H$ are

$$
\begin{aligned}
& \left(\delta_{l} e_{\gamma}\right)\left(\delta_{t} e_{\eta}\right)=\delta_{l \triangleleft \gamma, t} \sigma_{l}(\gamma, \eta) \delta_{l} e_{\gamma \eta}, \quad l, t \in L, \quad \gamma, \eta \in \Gamma, \\
& \Delta\left(\delta_{l} e_{\gamma}\right)=\sum_{u \in L} \tau_{\gamma}\left(u, u^{-1} l\right) \delta_{u} e_{\left(u^{-1} l\right) \triangleright \gamma} \otimes \delta_{u^{-1} l} e_{\gamma}, \quad l \in L, \quad \gamma \in \Gamma .
\end{aligned}
$$

Here $\delta_{l} e_{\gamma}:=\delta_{l} \otimes e_{\gamma} \in H$. Then the following holds:

- [K, Si, T1] $H$ is a Hopf algebra iff for all $x, y \in \Gamma, s, t \in L$.

(4.4) $\sigma_{s t}(x, y) \tau_{x y}(s, t)=\sigma_{s}(t \triangleright x,(t \triangleleft x) \triangleright y) \sigma_{t}(x, y) \tau_{x}(s, t) \tau_{y}(s \triangleleft(t \triangleright x), t \triangleleft x)$.

This happens e. g. if $\sigma$ and $\tau$ are trivial, case where we denote $H=\mathbb{k}^{L} \# \mathbb{k} \Gamma$. If (4.4) holds, then we have an exact sequence of Hopf algebras

$$
1 \longrightarrow \mathbb{k}^{L \hookrightarrow \stackrel{\iota}{\longrightarrow}} H \stackrel{\pi}{\longrightarrow} \mathbb{k} \Gamma \longrightarrow 1 \text {. }
$$

- AN, Theorem 2.10] gives necessary and sufficient conditions on $\sigma$ and $\tau$ so that $H=\mathbb{k}^{L} \tau_{\sigma_{\sigma}} \mathbb{k} \Gamma$ becomes a braided Hopf algebra with respect to a braiding $c: H \otimes H \rightarrow H \otimes H$. This braiding $c$ is uniquely determined by

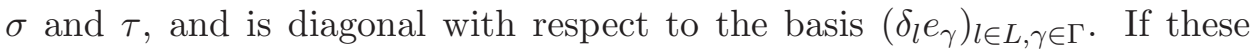
conditions hold, then $H$ can be realized as a braided Hopf algebra over an abelian group. We discuss this further in Subsection 4.3.

4.2.2. Automorphisms of an abelian extension with trivial cocycles. In subsection 4.2.3, we shall assume that $\sigma, \tau$ are trivial 2-cocycles. We shall give conditions on $H=\mathbb{k}^{L} \# \mathbb{k} \Gamma$ to be a $(G, \beta)$-color Hopf algebra, under the assumption that $A<$ Aut $_{\text {ext }} H:=\left\{f \in\right.$ Aut $H: f(x) \in \mathbb{k}^{L}$, for all $\left.x \in \mathbb{k}^{L}\right\}$. In this subsection, we determine Aut ext $H, H=\mathbb{k}^{L} \# \mathbb{k} \Gamma$. We first observe that this group is sometimes the full automorphism group.

Lemma 4.7. Then the following are equivalent:

(i) $\mathcal{H} Z(H)=\mathbb{k}^{L}$,

(ii) $\triangleleft$ is trivial and $Z(\Gamma)=\{1\}$.

If either of these conditions holds, then Aut $H=$ Aut $_{\text {ext }} H$.

Proof. (i) $\Rightarrow$ (ii): Since $\delta_{l, t} \delta_{l} e_{\gamma}=\delta_{l}\left(\delta_{t} e_{\gamma}\right)=\left(\delta_{t} e_{\gamma}\right) \delta_{l}=\delta_{t \triangleleft \gamma, l} \delta_{t} e_{\gamma}$, for all $l, t \in L$ and $\gamma \in \Gamma, \triangleleft$ is trivial. The subalgebra of $H$ generated by $\left\{\delta_{l} e_{\gamma}\right.$ : $l \in L, \gamma \in Z(\Gamma)\}$ is a central Hopf subalgebra of $H$, hence $Z(\Gamma)=\{1\}$.

(ii) $\Rightarrow$ (i): Clearly, $\pi(\mathcal{H} z(H))=\mathbb{k}$, because the former is a central Hopf subalgebra of $\mathbb{k} \Gamma$. Hence $\pi(y)=\varepsilon(y) 1$ for any $y \in \mathcal{H} z(H)$. Therefore, $\mathcal{H} \mathcal{Z}(H) \subseteq L \operatorname{Ker}(\pi)=\mathbb{k}^{L}$. Since $\triangleleft$ is trivial, $\mathbb{k}^{L} \subseteq \mathcal{H} \mathcal{Z}(H)$. The last assertion follows because the Hopf center is invariant under Aut $H$. 
Let $f \in$ Aut $_{\text {ext }} H$. If $l \in L$, then $f\left(\delta_{l}\right)=\delta_{f_{1}(l)}$, for some $f_{1}(l) \in L$; since $f$ is a automorphism of Hopf algebras, we see that $f$ induces $f_{1} \in$ Aut $L$. Analogously, $f$ induces $f_{2} \in$ Aut $\Gamma$, and we have a homomorphism of groups

$$
\Phi: \text { Autext }_{\text {e }} H \rightarrow \text { Aut } L \times \text { Aut } \Gamma, \quad \Phi(f)=\left(f_{1}, f_{2}\right) .
$$

Proposition 4.8. Let $(g, h) \in$ Aut $L \times$ Aut $\Gamma$. There exists $f \in$ Aut $_{\text {ext }} H$ such that $\Phi(f)=(g, h)$ if and only if the following conditions are satisfied:

(i) $g(l) \triangleleft h(\gamma)=g(l \triangleleft \gamma)$ and $g(l) \triangleright h(\gamma)=h(l \triangleright \gamma), \quad l \in L, \gamma \in \Gamma$.

(ii) There exists a map $\tilde{f}: \Gamma \rightarrow\left(\mathbb{k}^{\times}\right)^{L}, \gamma \mapsto \tilde{f}_{\gamma}: L \rightarrow \mathbb{k}^{\times}$(uniquely determined, see (4.15) ) such that

$$
\begin{aligned}
& \tilde{f}_{\gamma}(1)=1, \quad \gamma \in \Gamma, \\
& \tilde{f}_{1}(l)=1, \quad l \in L, \\
& \tilde{f}_{\gamma \eta}(l)=\tilde{f}_{\gamma}(l) \tilde{f}_{\eta}(l \triangleleft h(\gamma)), \quad l \in L, \quad \gamma, \eta \in \Gamma, \\
& \tilde{f}_{\gamma}(l t)=\tilde{f}_{g^{-1}(t) \triangleright \gamma}(l) \tilde{f}_{\gamma}(t), \quad l, t \in L, \quad \gamma \in \Gamma .
\end{aligned}
$$

If (i) and (ii) hold, then $f$ is given by

$$
f\left(\delta_{l} e_{\gamma}\right)=\widetilde{f}_{\gamma}(g(l)) \delta_{g(l)} e_{h(\gamma)}, \quad l \in L, \quad \gamma \in \Gamma .
$$

Proof. Let $f \in$ Aut $_{\text {ext }} H$ such that $\Phi(f)=(g, h)$. Set $\widetilde{e}_{\gamma}=1 \otimes e_{\gamma}, \gamma \in \Gamma$. Note that $f\left(\delta_{l} e_{\gamma}\right)=f\left(\delta_{l} e_{1} \cdot \widetilde{e}_{\gamma}\right)=\delta_{g(l)} f\left(\widetilde{e}_{\gamma}\right)$, for all $l \in L$ and $\gamma \in \Gamma$. Write

$$
f\left(\widetilde{e}_{\gamma}\right)=\sum_{\eta \in \Gamma} \sum_{t \in L} \widetilde{f}_{\gamma}(t, \eta) \delta_{t} e_{\eta}, \quad \widetilde{f}_{\gamma}(t, \eta) \in \mathbb{k} .
$$

Then $\sum_{\eta \in \Gamma} \widetilde{f}_{\gamma}(1, \eta) e_{\eta}=\pi \circ f\left(\widetilde{e}_{\gamma}\right)=h\left(e_{\gamma}\right)=e_{h(\gamma)}$, and consequently,

$$
\widetilde{f}_{\gamma}(1, \eta)=\delta_{h(\gamma), \eta}
$$

Given $\gamma, \tau \in \Gamma$, we have that

$$
\begin{aligned}
f\left(\widetilde{e}_{\gamma}\right) f\left(\widetilde{e}_{\tau}\right) & =\left(\sum_{u \in \Gamma} \sum_{m \in L} \widetilde{f}_{\gamma}(m, u) \delta_{m} e_{u}\right)\left(\sum_{v \in \Gamma} \sum_{n \in L} \widetilde{f}_{\tau}(n, v) \delta_{n} e_{v}\right) \\
& =\sum_{u, v \in \Gamma} \sum_{l \in L} \widetilde{f}_{\gamma}\left(n \triangleleft u^{-1}, u\right) \widetilde{f}_{\tau}(n, v) \delta_{n \triangleleft u^{-1}} e_{u v} ; \\
f\left(\widetilde{e}_{\gamma \tau}\right) & =\sum_{\eta \in \Gamma} \sum_{l \in L} \widetilde{f}_{\gamma \tau}(l, \eta) \delta_{l} e_{\eta}
\end{aligned}
$$

Hence

$$
\widetilde{f}_{\gamma \tau}(l, \eta)=\sum_{v \in \Gamma} \widetilde{f}_{\gamma}\left(l, \eta v^{-1}\right) \widetilde{f}_{\tau}\left(l \triangleleft \eta v^{-1}, v\right) .
$$




$$
\begin{aligned}
& \text { Also, } \Delta \circ f\left(\widetilde{e}_{\gamma}\right)=\sum_{\eta \in \Gamma} \sum_{r, t \in L} \widetilde{f}_{\gamma}(t, \eta) \delta_{r} e_{\left(r^{-1} t\right) \triangleright \eta} \otimes \delta_{r^{-1} t} e_{\eta} \text { and } \\
& \begin{aligned}
(f \otimes f) \circ \Delta\left(\widetilde{e}_{\gamma}\right) & =(f \otimes f)\left(\sum_{l \in L} \Delta\left(\delta_{l} e_{\gamma}\right)\right)=\sum_{l, s \in L} f\left(\delta_{s} e_{\left(s^{-1} l\right) \triangleright \gamma}\right) \otimes f\left(\delta_{s^{-1} l} e_{\gamma}\right) \\
& =\sum_{l, s \in L} \delta_{g(s)} f\left(1_{\mathbb{R} L} e_{\left(s^{-1} l\right) \triangleright \gamma}\right) \otimes \delta_{g\left(s^{-1} l\right)} f\left(\widetilde{e}_{\gamma}\right) \\
& \stackrel{\text { (4.11) }}{=} \sum_{\substack{u, v \in \Gamma \\
l, s \in L}} \widetilde{f}_{\left(s^{-1} l\right) \triangleright \gamma}(g(s), u) \widetilde{f}_{\gamma}\left(g\left(s^{-1} l\right), v\right) \delta_{g(s)} e_{u} \otimes \delta_{g\left(s^{-1} l\right)} e_{v} .
\end{aligned}
\end{aligned}
$$

This implies that

$$
\widetilde{f}_{\gamma}(r t, \eta)=\widetilde{f}_{g^{-1}(t) \triangleright \gamma}(r, t \triangleright \eta) \tilde{f}_{\gamma}(t, \eta) .
$$

It follows from (4.12) and (4.14) that $\tilde{f}_{\gamma}(r, \eta)=0$ whenever $\eta \neq h(\gamma)$. Consider the map $\tilde{f}: \Gamma \rightarrow\left(\mathbb{k}^{\times}\right)^{L}, \gamma \mapsto \widetilde{f}_{\gamma}$, given by

$$
\tilde{f}_{\gamma}(l)=\tilde{f}_{\gamma}(l, h(\gamma)), \quad l \in L .
$$

Then (4.6) and (4.8) follow, respectively, by (4.12) and (4.13). Also, (4.7) follows since $\sum_{l \in L} \delta_{l} e_{1}=1_{\mathbb{k}^{L}} e_{1}=f\left(1_{\mathbb{k}^{L}} e_{1}\right)=\sum_{l \in L} \widetilde{f}_{1}(l) \delta_{l} e_{1}$.

Using that $f\left(\delta_{l} e_{\gamma}\right)=\delta_{g(l)} f\left(\widetilde{e}_{\gamma}\right)=\widetilde{f}_{\gamma}(g(l)) \delta_{g(l)} e_{h(\gamma)}$, we obtain

$$
\begin{aligned}
f\left(\delta_{l} e_{\gamma} \cdot \delta_{t} e_{\eta}\right) & =\delta_{l \triangleleft \gamma, t} \widetilde{f}_{\gamma \eta}(g(l)) \delta_{g(l)} e_{h(\gamma \eta)}, \\
f\left(\delta_{l} e_{\gamma}\right) f\left(\delta_{t} e_{\eta}\right) & =\widetilde{f}_{\gamma}(g(l)) \widetilde{f}_{\eta}(g(t)) \delta_{g(l) \triangleleft h(\gamma), g(t)} \delta_{g(l)} e_{h(\gamma) h(\eta)} .
\end{aligned}
$$

The identity $g(l) \triangleleft h(\gamma)=g(l \triangleleft \gamma)$ is immediate from (4.16) and (4.17). Using that $(f \otimes f) \circ \Delta\left(\delta_{l} e_{\gamma}\right)=\Delta \circ f\left(\delta_{l} e_{\gamma}\right)$, we obtain that $g(l) \triangleright h(\gamma)=h(l \triangleright \gamma)$. Finally, the last identity and (4.14) imply (4.9).

Conversely, assume that (i) and (ii) hold. Define $f$ by (4.10); by a straightforward calculation, $f \in$ Aut $_{\text {ext }} H$ and $\Phi(f)=(g, h)$.

Remark 4.9. The conditions in Proposition 4.8 (ii) can be spelled out in cohomology terms. Indeed, the action $\triangleleft$ induces actions of $\Gamma$ on $\left(\mathbb{k}^{\times}\right)^{L}$ parameterized by $h \in$ Aut $\Gamma:\left(\gamma \rightarrow_{h} \phi\right)(l)=\phi(l \triangleleft h(\gamma)), \gamma \in \Gamma, \phi \in\left(\mathbb{k}^{\times}\right)^{L}$ and $l \in L$. Then (4.7) and (4.8) imply that $\tilde{f}$ is a normalized 1-cocycle. When $\triangleright$ is trivial, (4.6) and (4.9) imply that $\tilde{f} \in Z^{1}\left(\Gamma, \operatorname{Hom}\left(L, \mathbb{k}^{\times}\right)\right)$.

Example 4.10. Let $\Gamma=C_{3}=\langle\gamma\rangle$ and $L=C_{7}=\langle l\rangle$. Then $(\Gamma, L)$ is a matched pair with trivial $\triangleright$ and $\triangleleft: L \times \Gamma \rightarrow L, l \triangleleft \gamma=l^{2}$. Then $g \in$ Aut $L$, $g(l)=l^{-1}$, and $h=\mathrm{id} \in \operatorname{Aut} \Gamma$, satisfy the condition Proposition 4.8 (i). Let $1 \neq \xi \in \mathbb{k}$ such that $\xi^{7}=1$. The map $\tilde{f}: \Gamma \rightarrow \mathbb{k}^{L}, \widetilde{f}_{1}=1, \widetilde{f}_{\gamma}(l)=\xi$ and $\tilde{f}_{\gamma^{2}}(l)=\xi^{3}$, satisfies (4.6), (4.7), (4.8) and (4.9). By Proposition 4.8, there exists $f \in$ Aut $_{\text {ext }} H$ such that $\Phi(f)=(g, h)$. By (4.10), $f$ is given by

$$
f\left(\delta_{l^{i}} e_{1}\right)=\delta_{l^{-i}} e_{1}, \quad f\left(\delta_{l^{i}} e_{\gamma}\right)=\xi^{-i} \delta_{l^{-i}} e_{\gamma}, \quad f\left(\delta_{l^{i}} e_{\gamma^{2}}\right)=\xi^{-3 i} \delta_{l^{-i}} e_{\gamma^{2}} .
$$


Example 4.11. Let $\Gamma=C_{3}=\langle\gamma\rangle$ and $L=C_{12}=\langle l\rangle$. Then $(\Gamma, L)$ is a matched pair with the following actions

$$
\begin{array}{lll}
l^{2 k+1} \triangleright \gamma=l^{2 k} \triangleright \gamma^{2}=\gamma^{2}, & l^{2 k+1} \triangleleft \gamma=l^{2 k+5}, & l^{2 k+1} \triangleleft \gamma^{2}=l^{2 k+9}, \\
l^{2 k} \triangleright \gamma=l^{2 k+1} \triangleright \gamma^{2}=\gamma, & l^{2 k} \triangleleft \gamma=l^{2 k} \triangleleft \gamma^{2}=l^{2 k}, & l^{j} \triangleright 1=1 .
\end{array}
$$

Also $g \in$ Aut $L, g(l)=l^{7}$, and $h=\mathrm{id} \in \operatorname{Aut} \Gamma$, satisfy the condition Proposition 4.8 (i). Let $1 \neq \xi \in \mathbb{k}$ such that $\xi^{3}=1$. Then $\tilde{f}: \Gamma \rightarrow \mathbb{k}^{L}$, $\widetilde{f}_{1}=1, \widetilde{f}_{\gamma}\left(l^{2 k}\right)=\widetilde{f}_{\gamma^{2}}\left(l^{2 k}\right)=1, \widetilde{f}_{\gamma}\left(l^{2 k+1}\right)=\xi$ and $\widetilde{f}_{\gamma^{2}}\left(l^{2 k+1}\right)=\xi^{2}$, satisfies (4.6), (4.7), (4.8) and (4.9). By Proposition 4.8, there exists $f \in$ Aut $_{\text {ext }} H$ such that $\Phi(f)=(g, h)$. By (4.10), $f$ is given by

$$
\begin{aligned}
f\left(\delta_{l^{2 k}} e_{\gamma^{i}}\right) & =\delta_{l^{2 k}} e_{\gamma^{i}}, & f\left(\delta_{l^{2 k+1}} e_{1}\right) & =\delta_{l^{2(k+3)+1}} e_{1}, \\
f\left(\delta_{l^{2 k+1}} e_{\gamma}\right) & =\xi \delta_{l^{2(k+3)+1}} e_{\gamma}, & f\left(\delta_{l^{2 k+1}} e_{\gamma^{2}}\right) & =\xi^{2} \delta_{l^{2(k+3)+1}} e_{\gamma^{2}} .
\end{aligned}
$$

4.2.3. Color abelian extension. Let $\rho: A \rightarrow$ Aut $_{\text {ext }} H$ be a morphism of groups; we investigate when $H$ is a $(G, \beta)$-color Hopf algebra.

Given $a \in A$, we recall that there exists $\left(a_{1}, a_{2}\right) \in \operatorname{Aut} L \times \operatorname{Aut} \Gamma$ and $\tilde{a}_{\gamma}: \Gamma \rightarrow\left(\mathbb{k}^{\times}\right)^{L}$ such that $\Phi(a)=\left(a_{1}, a_{2}\right)$ and $a\left(\delta_{l} e_{\gamma}\right)=\tilde{a}_{\gamma}\left(a_{1}(l)\right) \delta_{a_{1}(l)} e_{a_{2}(\gamma)}$, $l \in L$ and $\gamma \in \Gamma$; see Proposition 4.8. We denote

$$
A_{\gamma}^{l}:=\left\{a \in A: a_{1}(l)=l, a_{2}(\gamma)=\gamma\right\}, G_{\gamma}^{l}:=\left\{g \in G: \chi_{g} \in A_{\gamma}^{l}\right\} .
$$

Clearly, the map $\varphi_{\gamma}^{l}: A_{\gamma}^{l} \rightarrow \mathbb{k}^{\times}, \varphi_{\gamma}^{l}(a)=\tilde{a}_{\gamma}(l)$, is a character of $A_{\gamma}^{l}$. Let $\chi_{\gamma}^{l}: G_{\gamma}^{l} \rightarrow \mathbb{k}^{\times}, \chi_{\gamma}^{l}=\varphi_{\gamma}^{l} \circ \chi$, and $\rho_{\gamma}^{l}: A \rightarrow \operatorname{Hom}\left(G_{\gamma}^{l}, \mathbb{k}^{\times}\right), \rho_{\gamma}^{l}(a)=\left.a\right|_{G_{\gamma}^{l}}$.

Definition 4.12. A $(G, \beta)$-color matched pair is a matched pair $(\Gamma, L)$ provided with a morphism of groups $\rho: A \rightarrow$ Aut $_{\text {ext }} H$ that satisfies: for all $u, l \in L$ and $\eta, \gamma \in \Gamma$,

(i) $\left(G_{\eta}^{u}\right)^{\perp}<A_{\gamma}^{l}$,

(ii) $A_{\gamma}^{l} \cap\left(\rho_{\eta}^{u}\right)^{-1}\left(\chi_{\eta}^{u}\right) \neq \emptyset$,

(iii) if $a \in A_{\gamma}^{l} \cap\left(\rho_{\eta}^{u}\right)^{-1}\left(\chi_{\eta}^{u}\right)$, then $\tilde{a}_{\gamma}(l)=1$.

Theorem 4.13. Let $(\Gamma, L)$ be a matched pair provided with a morphism of groups $\rho: A \rightarrow$ Aut $_{\text {ext }} H$. Then the following are equivalent:

(i) $H$ is a color Hopf algebra,

(ii) $\beta(g, h)=1$, for all $g, h \in \sup H$,

(iii) $(\Gamma, L)$ is a $(G, \beta)$-color matched pair.

Proof. (i) $\Leftrightarrow$ (ii) follows from Proposition 4.1. (ii) $\Rightarrow$ (iii): Let $l, u \in L, \gamma, \eta \in$ $\Gamma$ and $a \in\left(G_{\eta}^{u}\right)^{\perp}$. Since $\sup H \subset G_{\eta}^{u}, a \in A_{\gamma}^{l}$. Let $b \in A$ such that $\left.b\right|_{G_{\eta}^{u}}=\chi_{\eta}^{u}$ and fix $x=\delta_{l} e_{\gamma}=\sum_{g \in s(x)} x_{g}$. Since $s(x) \subset \sup H \subset G_{\eta}^{u}$, it follows that $b(g)=\left(\tilde{\chi}_{g}\right)_{\eta}(u)=1$, for all $g \in s(x)$. Then, $x=b(x)$ and consequently, $b \in A_{\gamma}^{l} \cap\left(\rho_{\eta}^{u}\right)^{-1}\left(\chi_{\eta}^{u}\right)$. Finally, if $c \in A_{\gamma}^{l} \cap\left(\rho_{\eta}^{u}\right)^{-1}\left(\chi_{\eta}^{u}\right)$, then $c(x)=x$. Hence, $\tilde{c}_{\gamma}(l)=1$. (iii) $\Rightarrow$ (ii) Let $g, h \in \sup H$. Consider $l, u \in L$ and $\gamma, \eta \in \Gamma$ such that $g \in s\left(\delta_{u} e_{\eta}\right)$ and $h \in s\left(\delta_{l} e_{\gamma}\right)$. If $k \in G_{\eta}^{u}$, then $\chi_{g^{-1}}(k)=\chi_{\eta}^{u}(k)$, that is, 
$\rho_{\eta}^{u}\left(\chi_{g^{-1}}\right)=\chi_{\eta}^{u}$. Let $a \in A_{\gamma}^{l}$ with $\rho_{\eta}^{u}(a)=\chi_{\eta}^{u}$. Then, $a \chi_{g} \in\left(G_{\eta}^{u}\right)^{\perp}$, which implies that $\chi_{g^{-1}} \in A_{\gamma}^{l}$. Thus, $1=\left(\tilde{\chi}_{g^{-1}}\right)_{\gamma}(l)=\chi_{g^{-1}}(h)=\beta(g, h)^{-1}$.

Example 4.14. Let $H$ and $f$ be as in Example4.11, $G=C_{2} \oplus C_{2}=\langle g\rangle \oplus\langle h\rangle$ and $\beta: G \times G \rightarrow \mathbb{k}^{\times}, \beta\left(g^{i}+h^{j}, g^{k}+h^{l}\right)=(-1)^{i l-j k}$. Then $A=\langle a\rangle \oplus\langle b\rangle$, where $a(g)=-1, a(h)=b(g)=1$ and $b(h)=-1$. Consider the action of $A$ on $H$ given by $a(u)=b(u)=f(u), a b(u)=u$, for all $u \in H$. Then $H$ is a color Hopf algebra since $\beta(x, y)=1$ for all $x, y \in \sup H=\{1, g+h\}$.

\subsection{Color abelian extensions with non-trivial braiding.}

Let $\sigma: \Gamma \times \Gamma \rightarrow\left(\mathbb{k}^{\times}\right)^{L}$ and $\tau: L \times L \rightarrow\left(\mathbb{k}^{\times}\right)^{\Gamma}$ be normalized 2-cocycles satisfying (4.1) and let $H=\mathbb{k}^{L} \tau_{\sigma_{\sigma}} \mathbb{k} \Gamma$ with product (4.2) and coproduct (4.3). In [AN, Section 3], the authors discuss when $\mathbb{k}^{L} \pi_{\sigma} \mathbb{k} \Gamma$ is a Hopf algebra in $\underset{\mathbb{R} G}{\mathbb{R} G} y \mathcal{D}$ with respect to an action and a coaction that are diagonal with respect to the basis $\left(\delta_{l} e_{\gamma}\right)_{l \in L, \gamma \in \Gamma}$; namely, they are determined by maps $z: L \times \Gamma \rightarrow G$ and $\omega: L \times \Gamma \rightarrow \operatorname{Hom}\left(G, \mathbb{k}^{\times}\right)$, by the rules

$$
g \cdot \delta_{l} e_{\gamma}=\omega(l, \gamma)(g) \delta_{l} e_{\gamma}, \quad \lambda\left(\delta_{l} e_{\gamma}\right)=z(l, \gamma) \otimes \delta_{l} e_{\gamma} .
$$

Since we are interested in the braided tensor category $\left(\operatorname{Vect}^{G}, c_{\beta}\right)$, it is enough to fix $z$ because $\omega$ is given by $\omega(l, \gamma)(g)=\beta(g, z(l, \gamma))$, see (1.6). Note that (4.2) and (4.3) are morphisms of $\mathbb{k} G$-comodules if and only if,

$$
\begin{aligned}
z(l, \gamma \eta)=z(l, \gamma) z(l \triangleleft \gamma, \eta), & l \in L, & \gamma, \eta \in \Gamma, \\
z(l t, \gamma)=z(l, t \triangleright \gamma) z(t, \gamma), & l, t \in L, & \gamma \in \Gamma .
\end{aligned}
$$

The following theorem is a consequence of [AN, Theorem 3.5].

Theorem 4.15. Suppose that $z: L \times \Gamma \rightarrow G$ satisfies (4.19) and (4.20). Then $H$ is a $(G, \beta)$-color Hopf algebra iff for all $l, t \in L$ and $\gamma, \eta \in \Gamma$,

$$
\begin{aligned}
& \sigma_{l t}(\gamma, \eta) \tau_{\gamma \eta}(l, t)=\beta(z(t, \gamma), z(l \triangleleft(t \triangleright \gamma),(t \triangleleft \gamma) \triangleright \eta)) \\
& \times \tau_{\gamma}(l, t) \tau_{\eta}(l \triangleleft(t \triangleright \gamma), t \triangleleft \gamma) \sigma_{l}(t \triangleright \gamma,(t \triangleleft \gamma) \triangleright \eta) \sigma_{t}(\gamma, \eta) .
\end{aligned}
$$

For the rest of this subsection, in order to determine maps $z$ satisfying (4.19) and (4.20), we assume that $\triangleright$ is trivial; this implies that $\triangleleft: L \times$ $\Gamma \rightarrow L$ is an action by group automorphisms. Then $\triangleleft$ induces left actions of $\Gamma$ on $\operatorname{Hom}(L, G)$ and on $\operatorname{Hom}(L, A)$; for instance, $(\gamma \rightarrow \phi)(l)=\phi(l \triangleleft$ $\gamma)$, for all $\gamma \in \Gamma, \phi \in \operatorname{Hom}(L, G)$ and $l \in L$. By [AN, Lemma 4.1], the correspondence $z \mapsto \widetilde{z}: \Gamma \rightarrow \operatorname{Hom}(L, G), \widetilde{z}(\gamma)(l)=z(l, \gamma), l \in L, \gamma \in \Gamma$, determines a bijection between the set of maps $z$ satisfying (4.19) and (4.20) and $Z^{1}(\Gamma, \operatorname{Hom}(L, G))$. We now look for $\sigma, \tau$ satisfying the compatibility condition (4.21). We consider the 2-cocycle $\tau$ as a map $\widetilde{\tau}: \Gamma \rightarrow Z^{2}\left(L, \mathbb{k}^{\times}\right)$ and the action of $\Gamma$ on $Z^{2}\left(L, \mathbb{k}^{\times}\right):(\gamma \cdot \varphi)(l, t)=\varphi(l \triangleleft \gamma, t \triangleleft \gamma), \gamma \in \Gamma$, $\varphi \in Z^{2}\left(L, \mathbb{k}^{\times}\right), l, t \in L$.

Theorem 4.16. Let $\widetilde{z} \in Z^{1}(\Gamma, \operatorname{Hom}(L, G))$ and suppose that the following compatibility condition holds

$$
\sigma_{l t}(\gamma, \eta)=\beta(\widetilde{z}(\gamma)(t), \gamma \rightarrow \widetilde{z}(\eta)(l)) \sigma_{l}(\gamma, \eta) \sigma_{t}(\gamma, \eta)
$$


Then $H$ is a $(G, \beta)$-color Hopf algebra if and only if $\widetilde{\tau} \in Z^{1}\left(\Gamma, Z^{2}\left(L, \mathbb{k}^{\times}\right)\right)$.

Proof. Immediate consequence of Theorem 4.15.

We end this subsection with examples of color Hopf algebras that are neither commutative nor cocommutative. As in [So, Chapter 3] we consider:

$$
\begin{array}{lr}
R \text { a finite ring, } & L=G=\text { the additive group of } R, \\
\Gamma \text { a finite group, } & \nu: \Gamma \rightarrow R^{\times} \text {a group homomorphism, } \\
\psi \in Z^{1}(\Gamma, L), & \phi \in Z^{2}(\Gamma, L), \\
\eta, \theta: L \rightarrow \mathbb{k}^{\times}, & \theta(l t u)=\theta(t l u), \forall l, t, u \in L, \\
l \triangleright \gamma=\gamma, \quad l \triangleleft \gamma=l \nu(\gamma), & l \in L, \quad \gamma \in \Gamma . \\
z: L \times \Gamma \rightarrow G, \quad z(l, \gamma)=l \psi(\gamma), & l \in L, \quad \gamma \in \Gamma, \\
\beta: G \times G \rightarrow \mathbb{k}^{\times}, \quad \beta(g, h)=\theta(g h)^{2}, & g, h \in G .
\end{array}
$$

Define $\sigma_{l}(\gamma, v)=\eta(l \phi(\gamma, v)) \theta\left(l^{2} \nu(\gamma) \psi(\gamma) \psi(v)\right)$, for $l \in L, \gamma, v \in \Gamma$. By [So, 3.3], $\widetilde{z}, \beta$ and $\sigma$ satisfy (4.22). By Theorem 4.16, $H$ is a $(G, \beta)$-color Hopf algebra for each $\widetilde{\tau} \in Z^{1}\left(\Gamma, Z^{2}\left(L, \mathbb{k}^{\times}\right)\right)$; see [AN], Lemma 4.7] for examples of such 1-cocycles $\widetilde{\tau}$ of $\Gamma$ in $Z^{2}\left(L, \mathbb{k}^{\times}\right)$.

Acknowledgments. This paper was done during a posdoctoral stage of the third author in the Universidad Nacional de Córdoba. He thanks N. Andruskiewitsch and I. Angiono for the kind hospitality.

\section{REFERENCES}

[A] N. Andruskiewitsch, Notes on extensions of Hopf algebras. Can. J. Math. 48, 3-42 (1996).

[AAY] N. Andruskiewitsch, I. Angiono and H. Yamane, On pointed Hopf superalgebras. Contemp. Math. 544, 123-140 (2011).

[AN] N. Andruskiewitsch and S. Natale, Braided Hopf algebras arising from matched pairs of groups. J. Pure App. Algebra 182, 119-149 (2003).

[AHS] N. Andruskiewitsch, I. Heckenberger and H.-J. Schneider, The Nichols algebra of a semisimple Yetter-Drinfeld module. Amer. J. Math. 132, 1493-1547 (2010).

[ARS] N. Andruskiewitsch, D. Radford and H.-J. Schneider, The Nichols algebra of a semisimple Yetter-Drinfeld module. J. Algebra 324, 2932-2970 (2010).

[AS] N. Andruskiewitsch and H.-J. Schneider, Pointed Hopf algebras. In "New directions of Hopf algebras", Math. Sci. Res. Inst. Publ. 43, 1-68 (2002).

[Ang] Angiono, I. Nichols algebras of unidentified diagonal type. Comm. Alg., to appear.

[BaP] Y. Bahturin and D. Pagon, Classifying simple color Lie superalgebras. Contemp. Math. 483, 37-54 (2009).

[BK] B. Bakalov and A. Kirillov Jr., Lectures on Tensor Categories and Modular Functors. Amer. Math. Soc. (2000).

[Bt] M. Beattie, Duals of pointed Hopf algebras. J. Algebra 262, 54-76 (2003).

[CPO] X.-W. Chen, T. Petit and F. V. Oystaeyen, Note on the cohomology of color Hopf and Lie algebras. J. Algebra 299, 419-442 (2006).

[CSO] X.-W. Chen, S. D. Silvestrov and F. van Oystaeyen, Representations and cocycle twists of color Lie algebras. Algebr. Represent. Theory 9, 633-650 (2006).

[DT] Y. Doi, M. Takeuchi, Multiplication alteration by two-cocycles - the quantum version. Comm. Alg. 22, 5715-5732 (1994). 
[Du] M. Durdević, Generalized braided quantum groups. Isr. J. Math. 98, 329-348 (1997).

[EG1] P. Etingof and S. Gelaki, Some properties of finite-dimensional semisimple Hopf algebras. Math. Res. Lett. 5, 191-197 (1998).

[EG2] - The classification of triangular semisimple and cosemisimple Hopf algebras over an algebraically closed field. Int. Math. Res. Not. 5, 223-234 (2000).

[H1] I. Heckenberger, Classification of arithmetic root systems. Adv. Math. 220, 59-124 (2009).

[H2] Lusztig isomorphisms for Drinfel'd doubles of bosonizations of Nichols algebras of diagonal type. J. Algebra 323, 2130-2180 (2010).

$[\mathrm{Hu}] \mathrm{N} . \mathrm{Hu}$, Quantum group structure associated to the quantum affine space, Algebra Colloq. 11, 483-492 (2004).

[K] G. I. Kac, Extensions of groups to ring groups, Math USSR Sb. 5, 451-474 (1968).

[KS] A. Klimyk and K. Schmügen, Quantum groups and their representations. Texts and Monographs in Physics. Springer-Verlag, Berlin, 1997. xx+552 pp.

[Ky] A. N. Koryukin, Color Lie superalgebras and Hopf algebras. Algebra Logic 34, 232-241 (1995).

[M2] S. Majid, Crossed products by braided groups and bosonization. J. Algebra 163, 165-190 (1994).

[Ma] A. Masuoka, Extensions of Hopf algebras. Trabajos de Matemática - Fa.M.A.F 41/99 (1999). Available at hhtp://www.mate.uncor.edu/ andrus.

[Mo] S. Montgomery, Constructing simple Lie superalgebras from associative graded algebras. J. Algebra 195, 558-579 (1997).

[N] W. D. Nichols, Bialgebras of type one. Comm. Algebra 6, 1521-1552 (1978).

[R] D. Radford, Hopf algebras with projection. J. Algebra 92, 322-347 (1985).

[RaS] D. Radford, H.-J. Schneider, On the simple representations of generalized quantum groups and quantum doubles. J. Algebra 319, 3689-3731 (2008).

[Re] R. Ree, Generalized Lie elements. Can. J. Math. 12, 493-502 (1960).

[Sch] P. Schauenburg, On the braiding on a Hopf algebra in a braided category. New York J. Math. 4, 259-263 (1998).

[S] M. Scheunert, Generalized Lie algebras. J. Math. Phys. 20, 712-720 (1979).

[ScZ] M. Scheunert and R. B. Zhang, Cohomology of Lie superalgebras and their generalizations. J. Math. Phys. 39, 5024-5061 (1998).

[Si] W. Singer, Extension theory for connected Hopf algebras. J. Algebra 21, 1-16 (1972).

[So] Y. Sommerhäuser, Yetter-Drinfel'd Hopf algebras over groups of prime order. Lect. Notes Math. 1789 (2002).

[T1] M. Takeuchi, Matched pair of groups and bismash product of Hopf algebras. Comm. Algebra 9, 841-882 (1981).

[T2] Finite Hopf algebras in braided tensor categories. J. Pure Appl. Algebra 138, 59-82 (1999).

[Y] H. Yamane, Representations of a $\mathbb{Z} / 3 \mathbb{Z}$-quantum group. Publ. Res. Inst. Math. Sci. 43, 75-93 (2007).

Famaf-Universidad Nacional de Córdoba, Ciem (COniCET), Medina Allende s/n, Ciudad Universitaria, 5000 Córdoba, República Argentina.

E-mail address: (andrus|angiono)@famaf .unc.edu .ar

Departamento de Matemática, Universidade Federal de Santa Maria, 97105900, SANTA MARIA, RS, BRAZIL

E-mail address: bagio@smail.ufsm.br 